\title{
An In-Depth Analysis Approach Enabling Precision Single Chain Nanoparticle Design
}

\author{
Johanna Engelke ${ }^{1,2,3}$, Bryan Tuten ${ }^{1,4 *}$, Ralf Schweins ${ }^{5}$, Hartmut Komber ${ }^{2}$, Leonie Barner ${ }^{1,6 *}$, Laura
} Plüschke ${ }^{2,3}$, Christopher Barner-Kowollik ${ }^{1,4 *}$, Albena Lederer ${ }^{2,3,7 *}$

\footnotetext{
${ }^{1}$ School of Chemistry and Physics, Queensland University of Technology, 2 George Street, Brisbane, QLD 4000, Australia

${ }^{2}$ Leibniz-Institut für Polymerforschung Dresden, Hohe Str. 6, 01069 Dresden, Germany

${ }^{3}$ School of Science, Technische Universität Dresden, 01062 Dresden, Germany

${ }^{4}$ Centre for Materials Science, Queensland University of Technology, 2 George Street, Brisbane, QLD 4000, Australia

${ }^{5}$ Institut Laue-Langevin, DS / LSS, CS 20 156, 71 Avenue des Martyrs, 38042 Grenoble CEDEX 9, France

${ }^{6}$ Institute for Future Environments, Queensland University of Technology, 4000 QLD Brisbane, Australia

${ }^{7}$ Stellenbosch University, Department of Chemistry and Polymer Science, Private Bag X1, Matieland 7602, South Africa
}

\begin{abstract}
The synthesis of single chain nanoparticles (SCNPs) is a vibrant field in macromolecular science, enabled by a rich variety of synthetic strategies to induce macromolecular chain folding. Due to the decrease of the hydrodynamic volume upon folding, SCNP formation is typically characterized by a shift towards higher elution volumes in size exclusion chromatography (SEC). However, a step-change in the methodologies for SCNP analysis is required for the in-depth understanding of the nature of intramolecular polymer folding and internal SCNP structure, which is critical to enable their application as catalytic nanoreactors. Herein, we exploit a unique combination of small-angle neutron scattering (SANS), ${ }^{19}$ F NMR spectroscopy, and quadruple detection SEC to generate an encompassing and systematic view of the folded morphology of poly(tert-butyl acrylate) based-SCNPs as a function of their reactive group density $(5,15$, and $30 \mathrm{~mol} \%)$ and absolute molar mass $(20,50,100 \mathrm{kDa})$. In addition to detailed morphological insights, we establish that the primary factor dictating the compaction of SCNPs is their reactive group density, which is ineffective below $5 \mathrm{~mol} \%$, reaching maximum compaction close to $30 \mathrm{~mol} \%$. The molar mass of the precursor polymers has a minor impact on how an SCNP compacts for molar masses above $20 \mathrm{kDa}$.
\end{abstract}

\section{Introduction}

The precisely folded structure of biomacromolecules, such as proteins and enzymes, dictates their biochemical function. ${ }^{1}$ Taking inspiration from nature, synthetic linear copolymers can be folded intramolecularly, forming dense single-chain nanoparticles (SCNPs). ${ }^{2,3}$ The intramolecular crosslinking can be of static or dynamic, non-covalent or covalent nature. ${ }^{4}$ The versatile molecular design of SCNPs allows for precise control over their architecture, ${ }^{5,6}$ conformation and size, ${ }^{7,8}$ rendering them ideal 
candidates for enzyme mimicry, ${ }^{9}$ advanced catalysis, ${ }^{10}$ drug delivery, ${ }^{11}$ or targeted imaging. ${ }^{12}$ Deducing generalized structure-property relationships for the size and conformation of SCNPs, regardless of the employed synthetic strategy, represents a fundamental step towards the advanced design of polymer nanoparticles, which is still lacking today. Yet, the essential requirement for the rational design of SCNP with properties close to the perfection of their natural counterparts is the fine control of the key parameters affecting SCNP formation. Thus, the precise analysis of the macromolecules before and after the folding process is critically required to establish complete understanding of these key parameters.

Analysis of the primary chemical structure of copolymers is conventionally carried out via ${ }^{1} \mathrm{H}$ and ${ }^{13} \mathrm{C}$ nuclear magnetic resonance (NMR) spectroscopy, while versatile NMR active nuclei enable monitoring the chemical transformations occurring during SCNP synthesis. A noteworthy illustration is a study that monitored the collapse of $\mathrm{Pt}^{\mathrm{II}}-\mathrm{SCNPs}$ by phosphine ligand coordination using ${ }^{31} \mathrm{P}\left\{{ }^{1} \mathrm{H}\right\} \mathrm{NMR}$ spectroscopy and corroborated via ${ }^{195} \mathrm{Pt}$ NMR measurements of the metal nuclei. ${ }^{13}{ }^{1} \mathrm{H}$ and DOSY NMR measurements was used by our team to monitor step-wise unfolding of SCNPs by changes in chemical composition, and hydrodynamic size. ${ }^{14}$ Furthermore, exploiting the high sensitivity of the ${ }^{19} \mathrm{~F}$ nucleus, Perez-Baena et al. studied successfully $\mathrm{B}\left(\mathrm{C}_{6} \mathrm{~F}_{5}\right)_{3}$ catalyzed folding reactions. ${ }^{15}$

Currently, size exclusion chromatography (SEC) is the most widely employed technique for mapping compaction during SCNP synthesis, as it separates molecules according to the hydrodynamic volume. The folding of SCNPs leads to a shift of the SEC trace towards higher elution volumes, relative to the starting material of the linear polymer (Figure 1 ). ${ }^{2}$ The assumption of relative size change is only valid under ideal SEC conditions, i.e. the separation occurs purely entropically and no enthalpic interaction with the columns occurs. ${ }^{2}$ Meeting these requirements is non-trivial, as the folding reaction usually entails chemical transformation. Thus, if the precursor did not experience enthalpic interactions with the stationary phase, the SCNP might behave differently, and vice versa. If the delay of the elution volume relative to a linear precursor is caused by both column interaction and hydrodynamic compaction of the analyte, the results will be significantly skewed.

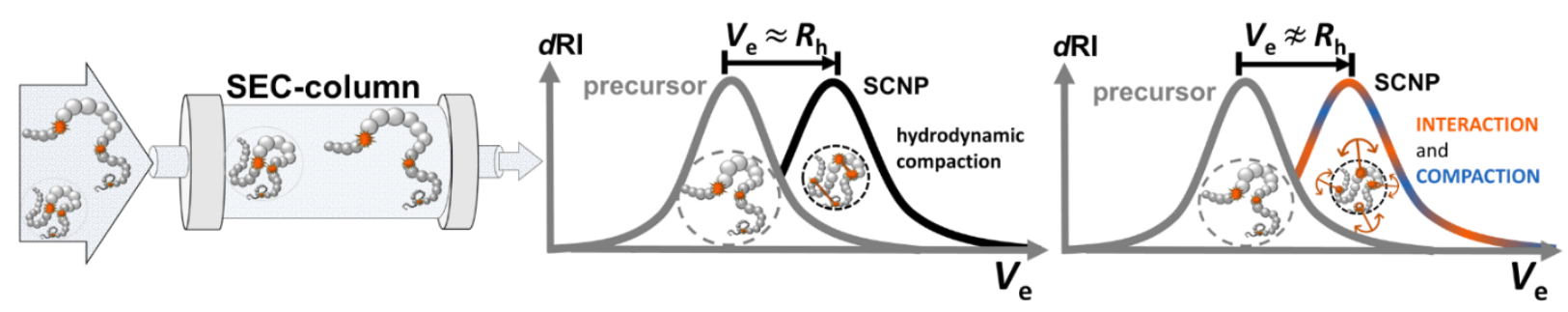

Figure 1. Following of SCNP formation by SEC: A shift of the SCNP trace in the chromatogram, relative to the starting material (precursor), is caused by hydrodynamic size reduction. Non-entropic separation processes caused by the chemical transformation of the functional groups during the folding reaction cannot be detected by simple concentration sensitive detectors like dRI. In this case, enthalpic interaction and compaction would interfere with the elution volume of the SCNPs.

The current limitation in monitoring the folding process of SCNPs relies on using SEC with simple differential refractometry (dRI) detection and calculation of sizes based on SEC standards. Even detection of absolute molar masses using multi-angle static light scattering (MALS) in SEC-dRI-MALS can be strongly limited due to the small sizes of SCNP structures. ${ }^{16}$ In order to gain deeper insight into the nature 
of SCNP folding, advanced hyphenated techniques such as hyphenating SEC-dRI-MALS systems to viscometry, fluorescence, or UV/Vis detectors are critically required. SEC-dRI-MALS enables the study of the molecular conformation of polymers and their apparent density, as well as revealing undesired multichain aggregation. ${ }^{17,18}$ Evaluation of the conformation via MALS requires a certain molar mass range (spanning at least two orders of magnitude), making narrowly distributed polymer samples difficult to analyze. ${ }^{2}$ In the case of isotropic scattering (SCNPs below $10 \mathrm{~nm}$ in diameter), or samples with a low optical contrast, the fundamental evaluation of the radius of gyration $\left(R_{\mathrm{g}}\right)$ and the absolute weight average molar mass $M_{\mathrm{w}}$ becomes impossible via MALS. Quasi-elastic light scattering (QELS, known also as dynamic light scattering) can determine the hydrodynamic radius $\left(R_{\mathrm{h}}\right)$ of particles down to only a few $\mathrm{nm}$, providing information on the segmental density and shape of the separated particles via the ratio $\rho=R_{\mathrm{g}} / R_{\mathrm{h}}{ }^{2}$ As aggregation or broad distributions lead to a drastic overestimation of the $R_{\mathrm{h}}$, QELS ideally should be coupled to a size separation technique (such as SEC). Unfortunately, the downstream dilution after SEC is an obstacle that SEC-QELS characterization of SCNPs has not overcome to date. By measuring rheological properties in solution, online viscometry (VS) is truly orthogonal to the optical detectors (QELS, MALS, $\mathrm{dRI}$ ), and therefore capable of analysing samples that isotropically scatter light, as well as samples with low optical contrast. The spatial compaction of SCNPs is indicated by a decrease in the intrinsic viscosity $[\eta]$, or the viscometric radius $\left(R_{\eta}\right)$, respectively. ${ }^{19-25}$ Viscosity data from SEC-dRI-MALS-VS experiments allows for the analysis of the solvent specific conformation of the polymer, providing valuable information about the macromolecular scaling or the solvent quality. Although limited techniques have been reported beyond SEC, complementary techniques to SEC separation such as mass spectrometry, ${ }^{26}$ atomic force microscopy (AFM) ${ }^{7,27-33}$ or cryogenic transmission electron microscopy (Cryo-TEM) ${ }^{21,34}$ enable detailed insights into the properties of SCNPs for appropriate materials.

Small-angle neutron scattering (SANS) provides unrivalled insights into soft matter materials. Due to the use of neutrons as the scattering source, SANS allows for non-destructive determination of the absolute radius of gyration $\left(R_{\mathrm{g}}\right)$ for particles between $1 \mathrm{~nm}-300 \mathrm{~nm}$ in size. ${ }^{35}$ A lot of information can be obtained from SANS data by a model-independent analysis, where standard plotting approaches such a Holtzer, Kratky, and Porod plots are the most commonly used. Furthermore, a model-dependent analysis can be performed by fitting the SANS data to various form factor models, which aids in deducing critical information regarding the microstructure (e.g. shape, mass fractals or segment density, compactness, stiffness of the polymer backbone, etc.), as long as a sufficient contrast is manipulated, e.g. by using either a (partially) deuterated solvent or by deuteration of parts of the solute. ${ }^{35}$ The disadvantages of SANS include high costs, the need for high sample quantity and purity, and extensive data-processing. However, its ability to provide characterisation in the sub $10 \mathrm{~nm}$ realm, which includes the majority of SCNPs, makes this an extremely powerful analytical tool. Initial neutron scattering characterization by Pomposo et al. provided insights into the shape and the compactness of SCNPs. ${ }^{36}$ Other studies have applied Kratky plots to the scattering data of SCNPs to confirm the increase of intramolecular density. ${ }^{36-40}$ Recently, a good prediction 
of experimentally observed relative chain collapses was set by a relation from a pool of literature values by invoking Flory-Fox theory. ${ }^{41}$

Theoretical calculation and simulation frequently assist the interpretation of experimental characterization. ${ }^{6,13,15,24,36,37,42-49}$ Chain-dynamics and collapse processes have been assessed, mostly by semi-empirical and statistical approaches. ${ }^{42}$ Not surprisingly, the complexity of macromolecular dynamics makes $a b$ initio quantum chemical calculations less applicable for SCNPs. Limiting the range of temperature and polymer lengths, Danilov et al. presented an extensive thermodynamic characterization of a reversible selective point folding, spanning the opening and closing transition with atomistic models. ${ }^{47}$ Importantly, Monte Carlo simulations enable the simultaneous tracing of the folding process. ${ }^{50}$ Very recently, Sommer and co-workers revealed the underestimated impact of solvent conditions during the folding on the SCNP structure..$^{51,52}$

Fundamental progress towards understanding the structure-property relationships of SCNPs has thrived in the last 10 years. Nevertheless, an in-depth understanding of the correlation between the key parameters molar mass and folding group density to the resulting SCNP morphology is critically missing. Thus, advanced characterisation techniques need to be developed to facilitate the purposeful design of SCNP structures. Herein, we fill this critical gap based on a unique combination of analytical techniques. The investigated SCNPs are constructed from poly(pentafluorobenzyl-stat-tert-butyl acrylate) precursors spanning a defined range of reactive group density (5 to $30 \mathrm{~mol} \%$ pentafluorobenzyl acrylate) and molar masses (20 to $100 \mathrm{kDa}$ ), and the resulting morphologies are investigated by a fusion of small-angle neutron scattering (SANS), ${ }^{19} \mathrm{~F}$ NMR spectroscopy and quadruple size-exclusion chromatography (SEC-RI-MALSVS-QELS), coined SEC-D4. This combination of sophisticated techniques provides an unprecedented picture of both the outer as well as inner structure of compactly folded single chains to deduce general trends of structure property relationships for any SCNP material.

\section{Results and Discussion}

The SCNP folding strategy in the current work is based on the regioselective para-fluoro thiol reaction (PFTR), which allows for the folding of the polymer precursors using an external dithiol crosslinker 1,4benzenedimethanethiol (BDMT). Inspired by the work of Roth et al., we employed pentafluorobenzyl acrylate (PFBA) as a functional monomer to introduce pentafluorobenzyl (PFB) moieties for the selective nucleophilic aromatic substitution at the para fluorine atom along the precursor backbone. ${ }^{53}$ Thiols exhibit increased acidity and nucleophilicity compared to amines or alcohols, allowing for less harsh reaction conditions for the regioselective PFTR reaction. ${ }^{54}$ Importantly, ${ }^{19} \mathrm{~F}$ NMR in combination with ${ }^{1} \mathrm{H}$ NMR spectroscopy facilitates the precise determination of the absolute SCNP composition, which we purposefully manipulated by molar mass and number of crosslinks of our defined precursor library (Figure 2). Thus, we tune the morphology of the SCNPs by both the systematic variation of the primary structure of precursor chains (repeat-unit folding approach), and of versatile length by a folding strategy in a poor solvent. ${ }^{55}$ 


\section{Linear Precursors}

The functional monomer pentafluorobenzyl acrylate (PFBA) was synthesized in a one-pot procedure described in the Supporting Information (SI, section 3.3).$^{53}$ The chain transfer agent cyanomethyl dodecyl trithiocarbonate (CMDT) facilitated the reversible addition-fragmentation chain transfer (RAFT) polymerization of the functional monomer pentafluorobenzyl acrylate (PFBA) and tert-butyl acrylate $\left({ }^{t} \mathrm{BuA}\right) .{ }^{56}$ Herein, three chain lengths of linear precursors with targeted molar masses of 20, 50, and 100 $\mathrm{kDa}$ were obtained. As depicted in Figure 2, each molar mass was synthesized for three different PFBA monomer contents ranging from low (approximately 5\%, colored green, A samples) to mid (approximately $15 \%$, colored blue, B samples) to high (approximately $30 \%$, colored black, C samples) feed ratios, randomly distributed per chain. As the amount of PFBA incorporation into the precursor backbone is in-line with the targeted values, the precursor library is suited for the fine-tuning of the ligation-density in the subsequent folding reaction.

Targeted library scheme

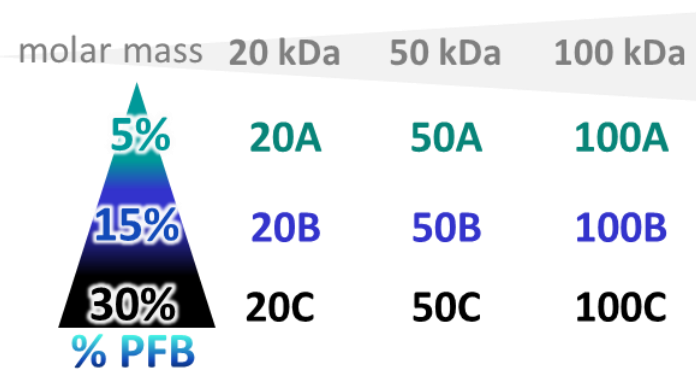

Structure

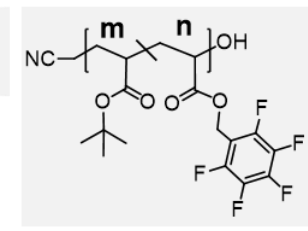

Experimental values

\begin{tabular}{ccccc}
\hline precursor & $\boldsymbol{M}_{\mathbf{n}}[\mathrm{kDa}]$ & $\boldsymbol{\oplus}$ & $\boldsymbol{m}$ & $\boldsymbol{n}$ \\
\hline 20A & 19.6 & 1.1 & 155 & 3 \\
20B & 19.5 & 1.1 & 114 & 19 \\
\hline 20C & 23.3 & 1.1 & 92 & 41 \\
50A & 59.2 & 1.1 & 395 & 19 \\
50B & 56.7 & 1.2 & 303 & 51 \\
50C & 54.9 & 1.2 & 216 & 86 \\
100A & 100.1 & 1.2 & 707 & 24 \\
100B & 105.0 & 1.4 & 643 & 102 \\
\hline 100C & 85.3 & 1.2 & 312 & 124 \\
\hline
\end{tabular}

Figure 2. Polymer library of the general structure depicted in the centre of the figure. The library scheme (left) shows the sample names denoting target molar mass (indicated by the number in the sample name) and PFB functionalisation (in mol\% indicated by the letter in the sample name; $5(A), 15(B), 30(C)$ ). Experimental data from SEC (absolute number average molar mass $M_{n}$ and polydispersity $Ð$ ) are given in the table. The number of repeating units, $n$ and $m$, are calculated from $M_{n}$, molar mass of repeating units and copolymer composition determined from ${ }^{1} \mathrm{H} N \mathrm{NR}$ spectra. Yellow background in the table indicates samples that were additionally characterized via SANS.

Initial SCNP folding of the RAFT-based precursors proved difficult due to the sulfur end groups, therefore, we employed a radical-induced oxidation in a facile one-pot procedure as described by Barner-Kowollik and coworkers in order to remove the terminal trithiocarbonate moieties. ${ }^{57-60}$ The optimization of the conditions of end group removal was performed on representative copolymers as described in the Supporting Information (section 3.4). The success of RAFT group removal was confirmed by ${ }^{1} \mathrm{H}$ NMR spectroscopy on smaller copolymers as evidenced by the disappearance of the $\mathrm{C}(\mathrm{S}) \mathrm{SCH}_{2}$ signal at $3.25 \mathrm{ppm}$ (section 3.4.2, SI-Figure 12). ${ }^{60,61}$ The quantification of the polymer composition was performed for both the parent RAFT copolymer, and for the precursor after trithiocarbonate group removal via ${ }^{1} \mathrm{H}$ NMR spectroscopy with only negligible deviation observed.

The composition of the polymer (comonomer ratio and crosslinker content for the SCNPs) per chain directly impacts the optical properties of the copolymers (refractive index, $\mathrm{d} n / \mathrm{d} c$, the extinction coefficient, etc.) and all concentration dependent measures, respectively. The determination of the optical contrast $\mathrm{d} n / \mathrm{d} c$ is critical for accurate molar mass determination using light scattering techniques and was determined for 
all precursors and the SCNPs. We employed a calculation procedure allowing to deduce reliable refractive index increments (Table 1) for any composition of the precursors as well as of the SCNPs (refer to section 3.2 of the SI).

\section{Single Chain Nanoparticles (SCNPs)}

The primary structure of the precursor was designed to enable a systematic variation in the final size and conformation of the SCNPs. The mol\% of PFB in the precursor polymers defines the reactive group density and therefore fine-tunes the number of crosslinks in the folding reaction. All crosslinkable moieties of the precursor as well as the procedure for the evaluation of the absolute SCNP composition, are depicted in Figure 3.
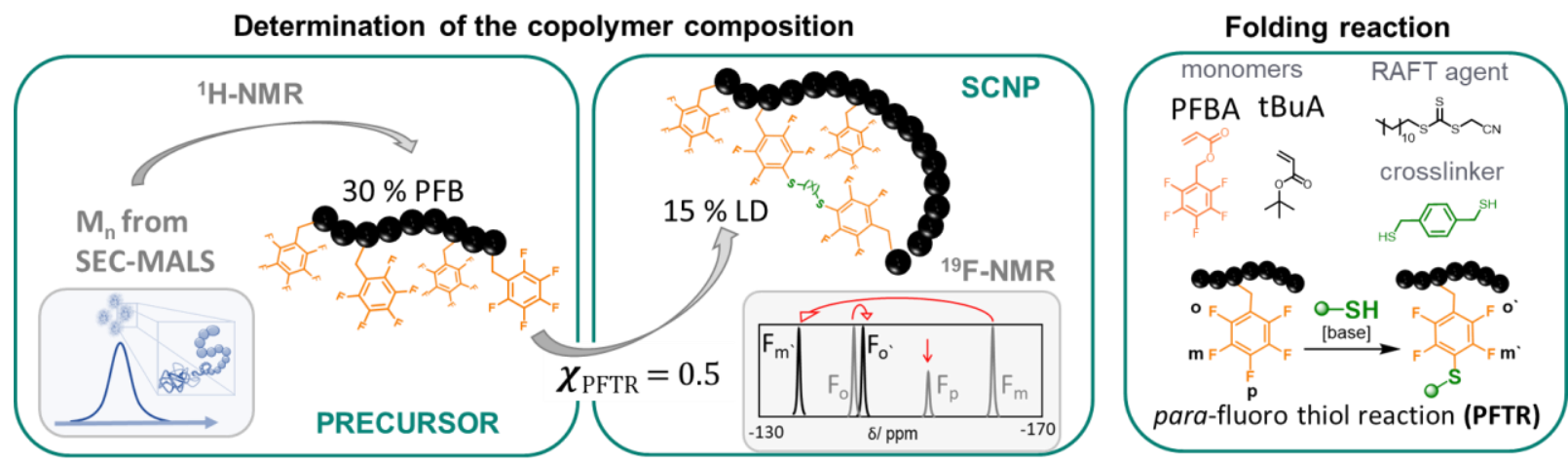

Figure 3. Synthetic strategy of the PFTR-based folding reaction (right box). Composition determination (left two boxes): The relative composition of the polymers is associated with an absolute number of building blocks for a given $M_{n}$ of the precursor and the molar mass of the building blocks. ${ }^{19} \mathrm{~F}$ NMR spectroscopy gives access to the amount of substituted para-fluorine atoms and the incorporated crosslinker under assumptions based on the distinct amount of PFB units in the precursor chains via ${ }^{l} H$ NMR spectroscopy.

Poor solvent conditions during the folding reaction, enabling the densest possible conformation of the linear precursor, are critical to generate compact SCNPs, as was demonstrated by Sommer et al. ${ }^{51}$ Optimal solubility of the precursor library was observed in polar solvents (i.e. poor solubility, below thetaconditions), facilitating a more densely packed random coil of the precursor polymer, thereby leading to a more tightly intramolecularly folded SCNP. Thus, the polar aprotic solvent acetonitrile (ACN) was ideal for the intramolecular folding process.

The external crosslinker 1,4-benzenedimethanethiol (BDMT) was added equimolarly with respect to the functional PFB groups of the precursor (refer to the SI, section 3.5). The use of an external crosslinker is analogous to substrate binding for enzyme activity - a diffusion governed process - thus a higher than stoichiometric amount of external crosslinker is required for successful intramolecular folding. ${ }^{62}$ Polar solvents not only facilitate the PFTR, but also the disulfide formation - a parasitic side reaction in this instance. ${ }^{63}$ To overcome this issue, a catalytic amount of dimethylphenylphoshine (DMPP) is utilized as a reducing agent to effectively avoid disulfide-linkage of the crosslinker. ${ }^{64,65}$ This parasitic side reaction would otherwise lead to decreased PFTR turnover and intermolecular ligation.

The successful generation of a structural hierarchy towards SCNPs, based on PFTR using an external crosslinker, required comprehensive optimization until a facile one-pot procedure was established (SI, 
section 3.5.1). Small additions of a good solvent (tetrahydrofuran, THF) was found to assist the PFTR reaction, whilst maintaining poor solvent conditions for the macromolecules. THF was added dropwise, under stirring, as the reaction rate of PFTR is rather slow in THF, ${ }^{63}$ until the solution became transparent. The required amount of good solvent was found to depend on the molar mass and on the amount of PFB incorporation. A clear dependence of the solvent quality with increasing PFB concentration (5\% PFB (A) $>15 \%$ PFB (B) > 30\% PFB (C)) in the polymer backbone of the precursor was observed. Highly PFB decorated precursors are less soluble because of the highly polar C-F bonds, which can alter the overall polarizability and solubility of highly fluorinated polymers. ${ }^{66-68}$ Excess of the base triethylamine (TEA) served not only to further solubilize the precursor, but also mitigated competitive behavior between the hydroxy endgroup of the precursor and the thiol-groups of the external crosslinker..$^{53,69-71}$ Attempts to increase the reaction rate by a stronger base were not successful. ${ }^{54,70-73}$ To compensate for the use of TEA and the dilution required for SCNP synthesis, long reaction times are required. ${ }^{63}$ The long reaction time is also intended to compensate for the increasing rigidity of the polymer as intramolecular ligation increases, and the decreasing of available PFB moieties. ${ }^{29}$ The folding reaction was quenched by scavenging the unreacted thiols via a Michael reaction with methylacrylate (MA), successfully avoiding intermolecular side reactions or column interaction during SEC characterization. ${ }^{63}$

The reaction times allowed the crosslinker molecules to reach even sparsely functionalized precursors in order to transform them into SCNPs at room temperature. Near quantitative conversion of the PFTR reaction was observed for the PFB groups of the $\mathrm{B}$ and $\mathrm{C}$ precursors, whereas the side-groups of sparcely decorated A-samples (approximately 5\% PFB) were transformed with approximately 50-60 \% PFTR conversion only (Figure 4). The lower reaction rate is in good correlation with the lower amount of reactive groups but additional reason is the more open precursor conformation, when compared to higher decorated samples of similar molar mass. ${ }^{46}$ Thus, the composition and the conformation of the precursor during the folding reaction are influencing the probability of folding events and the intra-chain distances, respectively. ${ }^{51}$

\section{Composition of the SCNPs}

The turnover of the intramolecular PFTR can be accurately quantified (refer to the SI, section 3.5.4) via highly sensitive ${ }^{19} \mathrm{~F}$ NMR spectroscopy. The pentafluorobenzyl moiety shows three distinct signals of the ortho-, meta-, and para-fluorine atoms as depicted in Figure 3 (refer to the SI, section 3.5.4) ${ }^{53}$ Whereas the integration of all signals $\left(F_{o}, F_{m}, F_{p}\right)$ enables the accurate quantification for small molecule experiments, only the signal integrals of the meta-flourine atoms in the precursor $\left(\mathrm{F}_{\mathrm{m}}\right)$ and in the reacted PFB moiety $\left(\mathrm{F}_{\mathrm{m}}\right)$ can be used to quantify the average conversion of the PFTR for polymers by

$$
\chi_{P F T R}=\left(F_{m \prime}\right) /\left(F_{m \prime}+F_{m}\right)
$$

To quantify the ligation events, we use the term of ligation density (LD). The LD is the number of crosslinking events of the functional side groups with respect to the degree of the polymerization of the precursor chain. When comparing different chain lengths, the relative value of the $\mathrm{LD}$ is a useful measure. 
Figure 3 shows that the amount of substituted para-fluoro atoms is a product of the PFTR-conversion $\chi_{P F T R}$ (determined via ${ }^{19} \mathrm{~F}$ NMR spectroscopy) and the molar amount of PFB units in the precursor's backbone (determined by ${ }^{1} \mathrm{H}$ NMR spectroscopy).

$$
L D=\left(\chi_{\text {PFTR }} * \% \mathrm{PFB}\right)
$$

We obtained a SCNP-library with approximately 3\% LD (A-samples), 14\% LD (B-samples) and 30\% LD (C-samples) of PFTR-converted side-groups (Figure 2 and Table 1).

Strictly speaking, crosslinking events cannot be derived from ${ }^{19} \mathrm{~F}$ NMR analysis of the PFTR reaction, since this only describes the reaction with the PFB moiety. The bifunctional crosslinker could potentially only be attached with one thiol (Figure 4). A crosslinking reaction has occurred only when both sides of the crosslinker have demonstrably intramolecularly reacted. According to this folding strategy, potentially oneside PFTR-reacted crosslinkers will be scavenged at the other thiol-group by methylacrylate (MA), ensuring that any residual free thiols will not form undesired intermolecular disulfide linkages upon concentration of the solution. Thus, the double-sided attachment of the crosslinker to the macromolecules must be confirmed. Due to signal overlay in the ${ }^{1} \mathrm{H}$ NMR spectra, direct quantification of the characteristic scavenger resonances is not necessarily indicative of the number of potential scavenging events. Instead, the ratio of the integral of the methylene group of the PFB-unit to the integral of the methylene groups of the crosslinker presented in Figure 4, can serve as a validation of the nature of the attachment of the crosslinker. As confirmed via the comparison of these ${ }^{1} \mathrm{H}$ NMR spectroscopic integrals to the $\chi_{\text {PFTR }}$ (Table in Figure 4), mainly two-side attached crosslinker molecules are present. Additionally, the scavenging scenario is negligible and the amount of incorporated bifunctional crosslinker molecules is equal to half of the ligation points, respectively.

As the relative composition of the polymer ultimately dictates absolute number of PFB moieties - given the knowledge of the absolute $M_{n}$ of the precursors and the molar mass of the PFBA monomer units - the average number of crosslinker per chain can be estimated for every sample. The average number of crosslinker per chain, equal to half of the number of the total number of ligation events per chain, increases with the total chain length for comparable LD. Comparison of the experimental SCNP molar masses (Figure 4 , left box, color coded) shows good agreement with the expected molar mass increase compared to the linear precursor (Figure 4, left box, red dots). Using SEC, we indicate an average molar mass increase of approximately 1-, 6-, and $12 \%$ for the A-, B-, and C precursor samples, respectively. These values are an important further confirmation of the primary structure of the SCNPs, i.e. an increase in molar mass but a decrease in size (see discussion below).

Thus, the obtained library of precursor-SCNP pairs serves well to deduce structure-property relationships with regard to the chain lengths and the amount of crosslinker units per chain (Table 1). The library is thus key for elucidation the size and conformation of the precursor and their corresponding SCNPs by SEC-D4 and SANS characterization. 


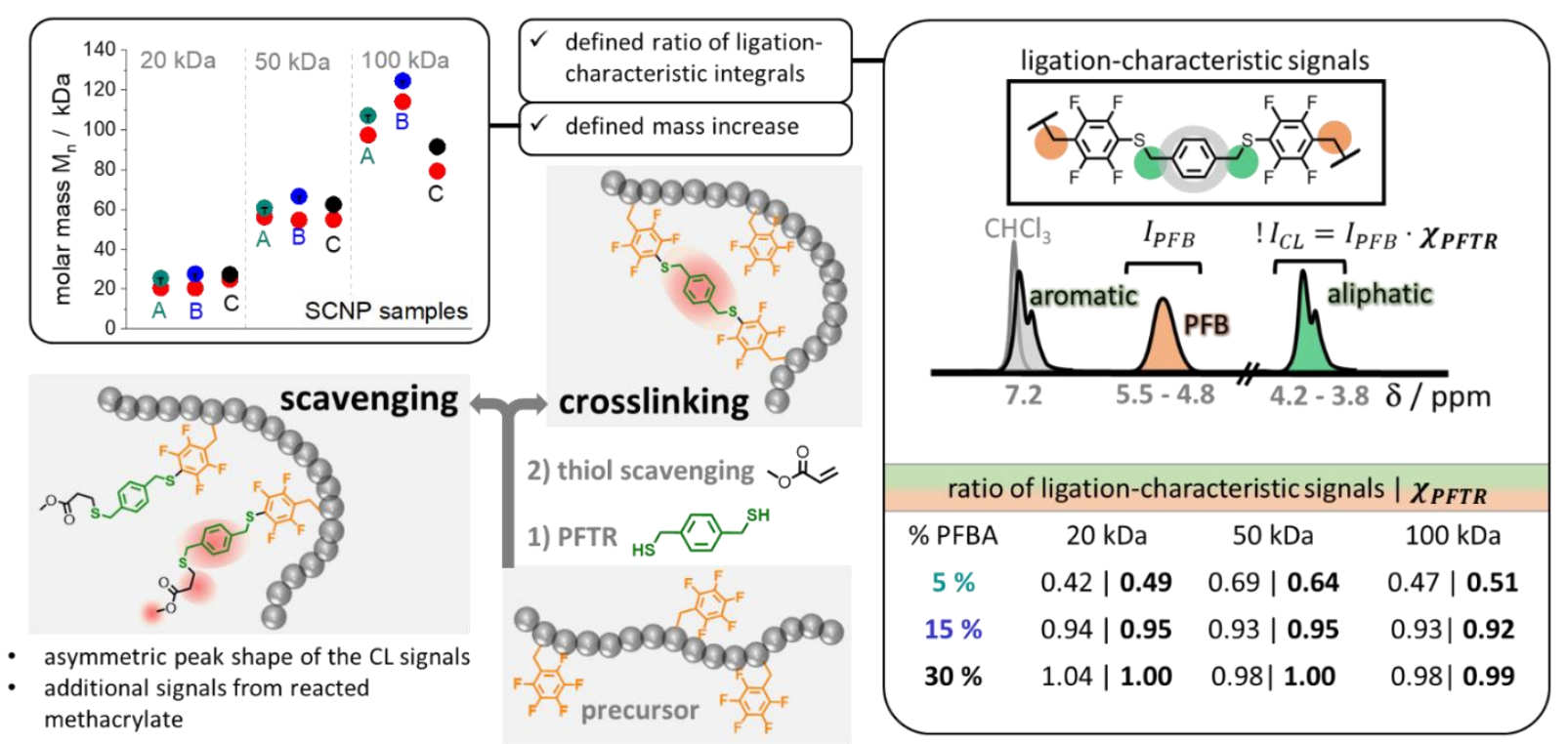

Figure 4. Possible products of the folding reaction and their validation using SEC-, and ${ }^{l} H$ NMR spectroscopy. Right box: The distinct ratio of the backbone-attached PFB methylene protons (orange) to the aliphatic protons of the crosslinker (green) allow to distinguish between loop-building or scavenged crosslinker. The aromatic resonances of the crosslinker (grey) are not suitable for this quantification due to overlap with the solvent resonances. The table shows the ratio of the peak areas of PFB signal (normalized to 1) to the aliphatic signals of the crosslinker. The ratio of ligation characteristic signals is approx. 0.5 (for $5 \%$ PFB with PFTR conversion 50\%) and 1.0 (15\% and 30\%PFB with PFTR conversion 100\%) confirming the PFTR conversion obtained from ${ }^{19} \mathrm{~F}$ NMR spectroscopy. This is additionally confirmed by the $M_{n}$-increase (left box) after incorporation of the corresponding number of crosslinker molecules (determined by NMR spectroscopy) from precursor (red dots) to SCNP (colour coded dots) obtained by SEC.

Table 1. Key parameters of the polymer library determined via ${ }^{1} \mathrm{H}$-, and ${ }^{19} \mathrm{~F}$ NMR spectroscopy, SEC-D4 and SANS (in THF,$T H F-d_{8}$ ). The full list of parameters and calculations is available in the SI Tables 8-10.

NMR

SEC-D4

SANS

\begin{tabular}{|c|c|c|c|c|c|c|c|c|c|c|c|}
\hline precursors & $\begin{array}{c}\text { PFB } \\
\%\end{array}$ & $D P^{\text {a) }}$ & $\boldsymbol{\boxplus}^{\mathbf{b})}$ & $\begin{array}{c}M_{w} \\
\mathrm{~kg}\end{array}$ & $\begin{array}{l}\boldsymbol{R}_{\boldsymbol{n}} \\
\mathrm{nm}\end{array}$ & $\alpha$ & $\begin{array}{l}\mathbf{d} \boldsymbol{n} / \mathrm{d} \boldsymbol{c} \\
\mathrm{mL} \mathrm{g}^{-1}\end{array}$ & $\begin{array}{c}\boldsymbol{R}_{g} \\
\mathrm{~nm}\end{array}$ & $v$ & 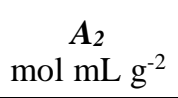 & $\begin{array}{c}\boldsymbol{l}_{\boldsymbol{K}} \\
\mathrm{nm}\end{array}$ \\
\hline $20 A$ & 2 & 158 & 1.09 & 22.5 & 3.8 & 0.56 & 0.052 & - & - & - & - \\
\hline $20 B$ & 14 & 133 & 1.10 & 21.3 & 3.5 & 0.55 & 0.051 & - & - & - & - \\
\hline $20 C$ & 31 & 133 & 1.07 & 23.8 & 3.4 & 0.59 & 0.050 & 5.1 & 0.6 & $2.11 \mathrm{E}-03$ & 3.2 \\
\hline $50 A$ & 5 & 414 & 1.18 & 65.5 & 7.0 & 0.60 & 0.052 & 11.6 & 0.5 & $2.04 \mathrm{E}-03$ & 3.6 \\
\hline $50 B$ & 14 & 354 & 1.24 & 63.8 & 6.8 & 0.59 & 0.051 & 10.9 & 0.6 & $1.35 \mathrm{E}-03$ & 4.0 \\
\hline $50 C$ & 29 & 302 & 1.22 & 60.2 & 6.1 & 0.57 & 0.050 & 9.8 & 0.6 & $8.65 \mathrm{E}-04$ & 4.2 \\
\hline $100 A$ & 3 & 731 & 1.21 & 116.6 & 9.9 & 0.64 & 0.052 & 14.9 & 0.5 & $-9.00 \mathrm{E}-04$ & 3.8 \\
\hline $100 B$ & 14 & 745 & 1.40 & 151.5 & 11. & 0.54 & 0.051 & - & - & - & - \\
\hline $100 C$ & 28 & 437 & 1.17 & 83.4 & 7.3 & 0.63 & 0.050 & 11.5 & 0.6 & $-1.48 \mathrm{E}-03$ & 3.7 \\
\hline SCNPs & $L D^{\mathrm{c})}$ & $C L^{d)}$ & & & & & & & & & \\
\hline$f 20 A$ & 1 & 1 & 1.15 & 29.3 & 4.4 & 0.50 & 0.054 & - & - & - & - \\
\hline$f 20 B$ & 14 & 9 & 1.17 & 32.4 & 3.6 & 0.37 & 0.072 & - & - & - & - \\
\hline$f 20 C$ & 31 & 21 & 1.11 & 30.4 & 3.3 & 0.31 & 0.097 & 3.6 & 0.4 & $-1.42 \mathrm{E}-03$ & - \\
\hline$f 50 A$ & 3 & 6 & 1.18 & 72.0 & 6.5 & 0.58 & 0.057 & 10.1 & 0.5 & $-7.06 \mathrm{E}-04$ & - \\
\hline$f 50 B$ & 14 & 24 & 1.20 & 79.9 & 5.4 & 0.35 & 0.072 & 7.3 & 0.3 & $-6.17 \mathrm{E}-04$ & - \\
\hline$f 50 C$ & 29 & 43 & 1.23 & 77.1 & 4.7 & 0.22 & 0.094 & 6.0 & 0.3 & $-1.22 \mathrm{E}-04$ & - \\
\hline f100A & 2 & 6 & 1.19 & 127.3 & 9.3 & 0.61 & 0.055 & 14.1 & 0.5 & $-1.19 \mathrm{E}-03$ & - \\
\hline$f 100 B$ & 13 & 47 & 1.41 & 176.1 & 8.1 & 0.31 & 0.070 & - & - & - & - \\
\hline flooC & 28 & 62 & 1.21 & 111.2 & 5.5 & 0.22 & 0.093 & 7.0 & 0.3 & $1.05 \mathrm{E}-04$ & - \\
\hline
\end{tabular}

a) degree of polymerisation; ${ }^{\mathbf{b})}$ molar mas distribution $Ð=M_{w} / M_{n}$; ${ }^{\mathbf{c})} \mathrm{LD}$ in $\%$; ${ }^{\mathbf{d})}$ crosslinks per chain 


\section{Molar mass, size and conformation via advanced size exclusion chromatography}

State-of-the-art SEC-D4 characterization enables a comprehensive analysis of the precursor library and their folded counterparts. The successful interpretation of the SEC-D4 experiments depends on the precise determination of $\mathrm{d} n / \mathrm{d} c$ (Eqs. SI-2 and SI-3), which is challenging in case of copolymers with composition variation. Very low optical contrast was observed for the precursor library in THF $(\mathrm{d} n / \mathrm{d} c$ approx. 0.05 $\mathrm{mL} / \mathrm{g}$, see Table 1). Such an observation is somewhat expected due to the highly polar C-F bonds decreasing the overall polarizability of multi-fluorinated organic molecules, thus, influencing the refractive index increment $\mathrm{d} n / \mathrm{d} c{ }^{66-68}$ In contrast, sulphur belongs to the group of highly polarizable main group elements and the C-S bond has low polarity. ${ }^{74}$ Therefore, the refractive index of the SCNPs is significantly increased due to incorporation of the dithiol-crosslinker. A comprehensive strategy for reliable evaluation of $\mathrm{d} n / \mathrm{d} c$ dependent on the varied copolymer composition for both precursor and SCNPs is provided in the SI section 3.2. The exact knowledge of $\mathrm{d} n / \mathrm{d} c$ is critically needed for samples of non-quantitative mass-recovery during SEC separation, which by definition limits the precise online determination of the $\mathrm{d} n / \mathrm{d} c$. Quantitative massrecovery in SEC separation for all precursors and most SCNPs (SI Table 8) allowed for reliable SEC interpretation and comprehensive online analysis of the $\mathrm{d} n / \mathrm{d} c$ values of the complete library.

Figure 5a shows the dRI traces from the SEC-D4 separation for the $50 \mathrm{kDa}$ samples (complete data in SI, section 4.2), including the molar mass and the intrinsic viscosities of the precursors and their folded analogues. Initial observation of the dRI traces shows monomodal distribution for the starting material, as well as for the SCNPs. The small high molar mass shoulder in some chromatograms is most probably caused by backbiting as a side reaction. ${ }^{75,76}$ This shoulder disappears after folding due to further precipitation procedures. Table 1 shows that the overall polydispersity $\oslash$ is not significantly altered after the folding, indicating that the purification after the folding compensates for the generally expected increase of polydispersity during the folding process. ${ }^{77}$ The shift of the peak from the precursor to the corresponding SCNP reveals the apparent decrease of the hydrodynamic size, which is more pronounced for higher LDs of the SCNPs. This observation is directly supported by the change of the intrinsic viscosity from the precursor to the corresponding SCNP (Figure 5a). Figure 5b indicates the direct correlation between the extent of apparent hydrodynamic collapse by the peak apex shift along the elution volume axis $\left(\Delta V_{e}\right)$ to the number of crosslinks per chain for all samples. Comparing the samples with similar LD (Figure 5c), it is obvious that the apparent hydrodynamic collapse is more significant for longer chains, because they possess larger number of segments, which increases the freedom for stronger conformational changes as discussed below. 

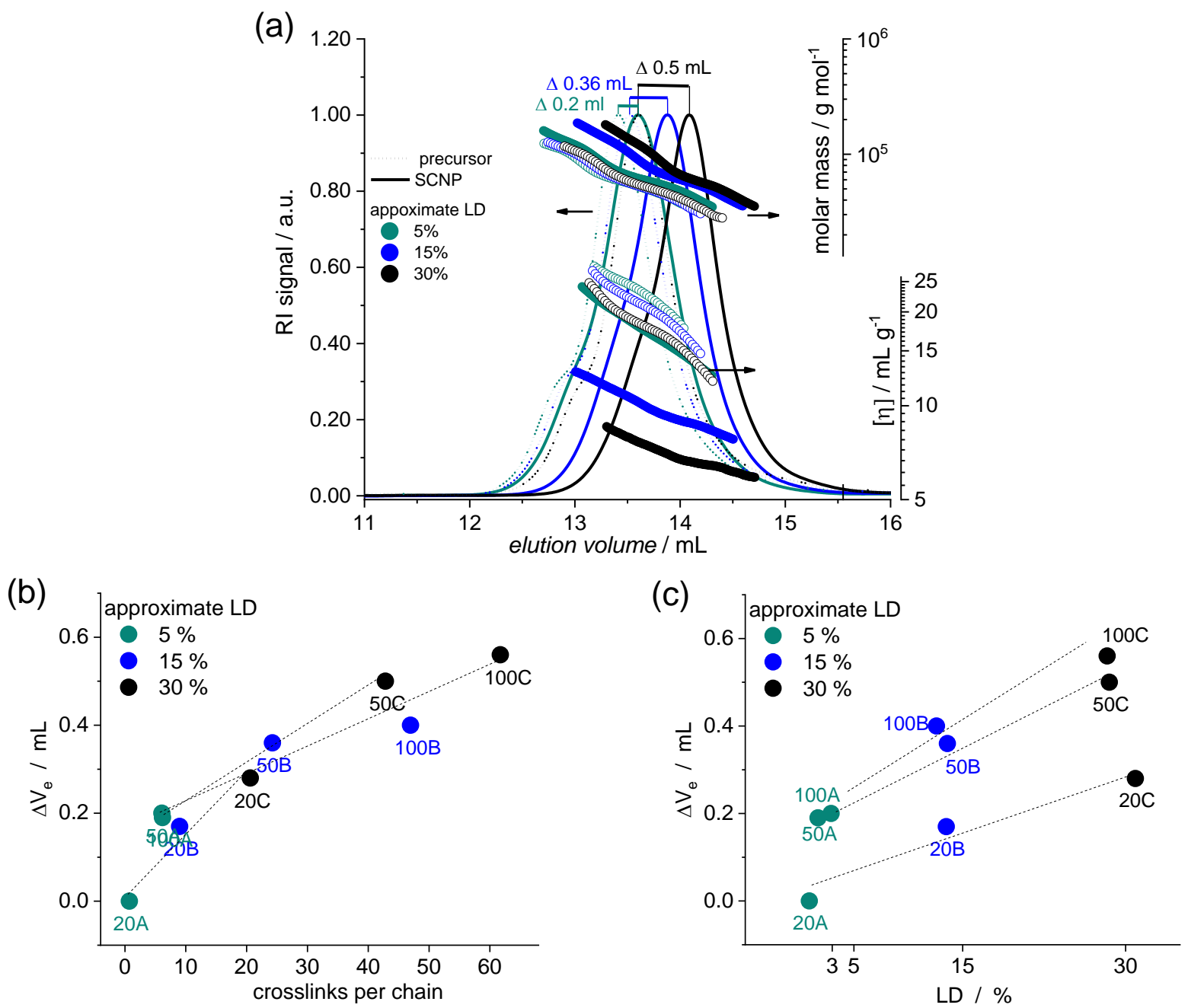

Figure 5. SEC-D4 results of the $50 \mathrm{kDa}$ samples as an example for the trends observed for the sample series (100 kDa-, and 20 kDa data are provided in the SI section 4). (a) dRI signal showing the shift towards higher elution volumes, a decrease of intrinsic viscosity [ $\eta]$ due to compaction after folding and an increase of molar mass due to crosslinker incorporation (compare Figure 4). These trends confirm successful transformation from the precursor (open dots and dotted dRI line) to their folded analogues (full dots and full dRI line). (b) Estimated shift of the elution volume $\Delta V_{e}$ of SCNPs with respect to the unfolded copolymer in dependency on the absolute number of crosslinks per chain. (c) Estimated shift of the elution volume $\triangle V_{e}$ of SCNPs with respect to the unfolded copolymer in dependency of $L D$.

Hyphenation of SEC to dRI and MALS detectors allows for evaluation of the absolute molar mass of the macromolecules (theoretical background is given in the SI section 1). Additional interpretation of the angular dependence of the scattering intensity enables the determination of the radius of gyration $\left(R_{g}\right)$. The dependence between $R_{g}$ and the molar mass delivers the scaling exponent $v$ according to Eq. (3).

$$
R_{g}=K M^{v}
$$

Unfortunately, the determination of $R_{g}$ is limited for particle sizes smaller than $\lambda / 20 \mathrm{~nm}$ (with $\lambda$ the wavelength of the incident beam) because of the lack of angular dependence of their scattering intensity (isotropic scattering). With size ranges of below $12 \mathrm{~nm}$, the SCNPs fall within the isotropic scattering regime. Even though, the reproducibility of the triple determination of the $R_{g}$ values for the $50 \mathrm{kDa}-$, and the $100 \mathrm{kDa}$ samples is high (SI Table 8), it is difficult to access $R_{g}$-molar mass dependence: The reliable 
determination of the scaling parameter $v$ from MALS requires a spanning of at least two orders of magnitude of molar mass range, making narrow distributions challenging. ${ }^{2}$ Nevertheless, absolute molar mass determination was successfully performed and a steady decrease of the molar mass with increasing elution volume was observed indicating no significant influence of enthalpic interactions on the SEC results. The increase of molar mass from precursors to corresponding SCNP (Figure 5a and Table 1) clearly correlates to the theoretical amount of incorporated crosslinker $(\mathrm{C}>\mathrm{B}>\mathrm{A})$, confirming the success of the folding reaction.

As discussed in the introduction, QELS is a complementary approach to MALS for determination of small polymers down to several $\mathrm{nm}$. Therefore, part of the SEC-D4 equipment was a QELS-detector for the determination of the hydrodynamic radius $R_{h}$. According to the Stokes-Einstein equation (Eq. SI-6) $R_{h}$ corresponds to the radius of a hypothetical sphere which possesses the same diffusion coefficient as the measured macromolecule. $R_{h}$ was frequently used for investigation of SCNPs using QELS in batch. ${ }^{15,78-80}$ Yet, the scattering intensity is proportional to the radius to the $6^{\text {th }}$ power, leading to overestimation of the radii whenever impurities (dust or broad distributions) are present in the sample. The limited precision of QELS-batch characterization was analysed by our team, noting that it can lead to strong deviation from reality. ${ }^{80}$ Consequently, QELS detection is most effective when coupled to a size separation, as each elution fraction can be considered monodisperse. ${ }^{18}$ Unfortunately, poor signal-to-noise ratio is a well-known obstacle due to dilution in the SEC column additionally to the low optical contrast $\mathrm{d} n / \mathrm{d} c$. Therefore, only the $R_{h}$ from samples with sufficiently high $\mathrm{d} n / \mathrm{d} c$ (SCNP with high crosslinker incorporation) were successfully characterized via online-QELS technique and are listed in SI Table 9. In conclusion, due to the limits of the light scattering, the reliable analysis of $R_{g}$ and $R_{h}$ was not possible over the complete range of samples' library via SEC-D4.

Online viscometry is orthogonal to all previously described detectors, as it measures rheological properties in solution and is independent of their optical features. In contrast to the results from MALS and QELS detectors, the signal of the viscometer shows excellent signal-to-noise ratio - even for particle sizes below the size-limit of light scattering. The spatial compaction of SCNPs should lead to a decrease in intrinsic viscosity $[\eta]$ which in turn is correlating with a decrease in the viscometric radius, $R_{\eta}$, according to Eq. (4).

$$
[\eta]=\frac{10 \pi}{3} N_{A} \frac{R_{\eta}^{3}}{M}
$$

Although the methods for determination of $R_{h}$ and $R_{\eta}$ are based on different physical principles, both radii are derived under the assumption of a sphere equivalent and as a fact, it was frequently found that their values are in a good agreement. ${ }^{81}$ Viscometric radii between 3 and $15 \mathrm{~nm}$ are obtained for the linear precursors (Table 1) with a trend of increasing average $R_{\eta}$ with the molar mass as expected (Figure 6a). 

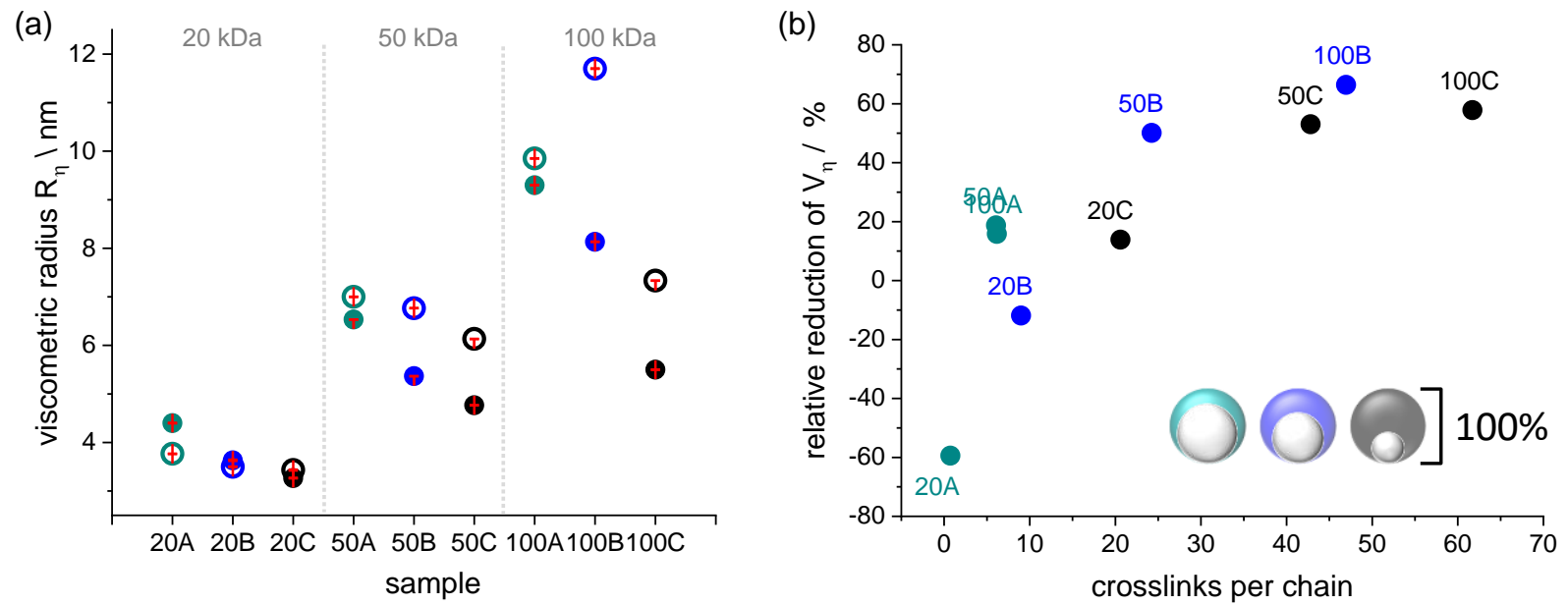

Figure 6. (a) Viscometric radii $R_{\eta}$ of the precursor (open dots) and the SCNPs (full dots), ordered with increasing molar mass (see grey labels as guide for the eye) and $L D$ from left to right. The error bars indicate high reproducibility of the results. (b) The reduction of the volume $\Delta V_{\eta} / V_{\eta, p r e c u r s o r}$ (based on $R_{\eta}$ and assuming spherical particles). Very small polymers (20 kDa samples) show unexpectedly low values, presumably due to anisotropic (2D) folding, which is the result of poor solvent conditions and short segment lengths.

Assuming spherical polymer shapes, statements about the 3D-contraction relative to the precursors volume are possible. The relative reduction of $V_{\eta}$, calculated from $R_{\eta}$ (Figure $6 \mathrm{~b}$ ), shows only $20 \%$ volume change for the less PFB decorated A-samples, whereas the stronger PFB decorated B-, and C-samples show up to $70 \%$ volume contraction.

The SCNPs of all molar mass ranges clearly follow the expected trend that increasing number of crosslinks per chain lead to increasing chain collapse of the SCNPs and confirm the volume reduction found by the shift of $V_{e}$ (Figure $\left.5 \mathrm{~b}, \mathrm{c}\right)$.

In contrast to the $50 \mathrm{kDa}$ and $100 \mathrm{kDa}$ samples, we obtain atypical results for the smallest (20 kDa) samples (Figure 6b). Sample 20A shows a slight increase of $R_{\eta}$, in contrast to the expected decrease as found for 20C. This unexpected result is unlikely associated with experimental error as the triple determination of $R_{\eta}$ for all 18 samples shows not only a low uncertainty for each experiment, but is also reproducible for each sample (SI Table 9). Nevertheless, it is not possible to suggest a lower physical limit for small particle size of this technique, as it strongly depends on the pressure, the temperature and the set-up.

We hypothesize that the short chain length is to be the reason for the atypical size development of the 20 $\mathrm{kDa}$ samples resulting in smaller volume reduction compared to larger precursor chains. Only short distance interactions (i.e. more local crosslinking as opposed to end-to-end crosslinking) are possible in shorter chains, while longer chains have greater potential for long-distance interactions between the macromolecular segments. This is justified with the small number of segments at a fixed persistence length. Figure 7 shows the effect of the polymerisation degree on the degree of compaction, which is even more pronounced for poor solvent conditions. While characterisation is carried out in good solvent conditions in the stages before and after the folding reaction (Figure 7, green modes), poor solvent conditions are chosen for the folding process (Figure 7, red mode). 


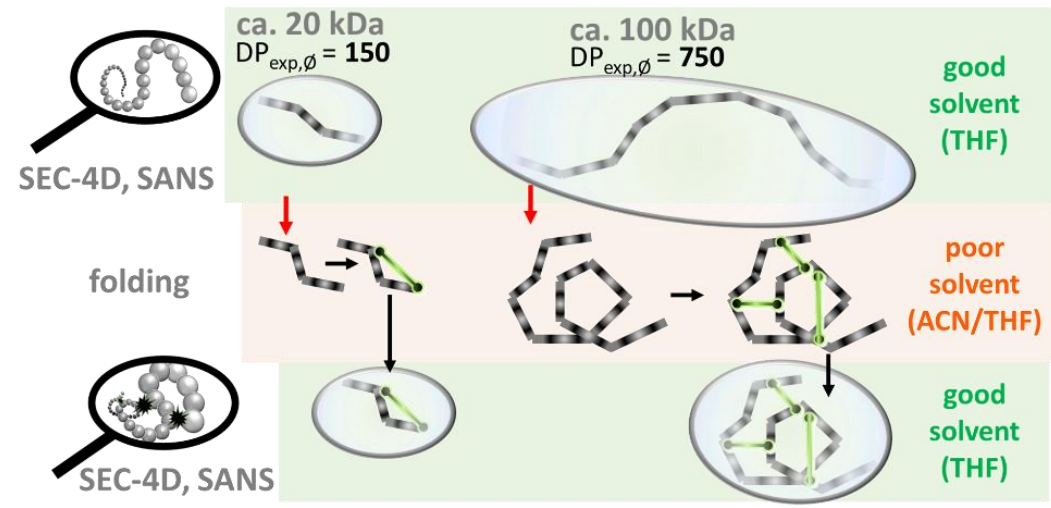

Figure 7. Schematic representation of the folding process in a poor solvent showing the lowest and the highest degree of polymerization DP exp,ø. Lack of or marginal chain collapse is shown for very small SCNPs, whereas significant collapse occurs for the large polymers. This chain collapse is justified by SEC-D4 and SANS characterization of the material prior and after the folding in a good solvent. In contrast, poor or close to theta-solvent conditions are adjusted during the folding.

The reason for this difference is that high dilutions and the used binary solvent required during folding are not ideal for the clear interpretation of analytical experiments. Furthermore, the characterization of the starting material in a good solvent represents the state of statistical linear chains. However, it should be noted that while the larger polymerisation degrees lead to conformations close to ideal coil statistics with pronounced fractal behaviour, the lower polymerisation degrees are excluded from this behaviour and have conformations closer to worm-like chains. Thus, the change to poor solvent conditions (Figure 7, red arrows) close to the theta-state leads to a more compact precursor conformation when the polymerisation degree is sufficiently high, increasing the probability of self-interaction to foster a tight intramolecular ligation (Figure 7, red mode). The base-induced intramolecular ligation (Figure 7, black arrows) can be referred to as a 'covalent freeze' of the chain conformation under these conditions, giving the quality of the solvent a paramount meaning for the final SCNP conformation. ${ }^{11}$ The subsequent characterization of the SCNPs in a good solvent mirrors the dominant morphology of the precursors under the folding conditions.

Of course, such behaviour depends on the number of crosslinks per segment which is reflected in the relative numbers of crosslinks per precursor chain (or LD in \%). The trend of increasing compaction is similar for all molar mass series (see Figure 5c). However, the effect of the absolute amount of crosslinks per chain seems to be the driving parameter of the folding behavoiur and is depending on the contour length of the chains. Furthermore, having a particular composition and thus, particular persistence length of the macromolecule implies an increasing number of the segments (increasing molar mass) will lead to increasing number of long-distance interactions between the segments for long chains. In contrast, predominantly short distance interactions occur for shorter chains, which may rather occur along the chain (2D contraction) than over intra-chain space (3D contraction, according to the classical understanding of folding). Further experimental confirmation of other repeat unit folding SCNP-systems is beneficial to validate this hypothesis of the interplay between chain-length and folding conditions.

Information on the compactness of polymer chains is obtained by the molar mass dependence of the intrinsic viscosity according to the Kuhn-Mark-Houwink (KMH) plot, which by theory correlates with the 
scaling law (Eq. 3). The KMH plot is shown for the $50 \mathrm{kDa}$ sample-set (50A, 50B, 50C) in Figure 8a. Significant change of the slope in the $\log [\eta] v s . \log M$ plot indicates the change of the macromolecular conformation from precursor to the folded analogue according to Eq. (5).

$$
[\eta]=K M^{\alpha}
$$

The average KMH-slopes of all samples are given in Table 1 and in Figure 8. All precursors show KMHslopes above 0.5 , corresponding to a polymer coil in good solvent conditions. ${ }^{35}$ Lower values indicate a more compact structure for the corresponding SCNPs. The SCNPs of the lowest LD (A-samples) show no significant change of compactness, as the KMH-slope only changes marginally. In contrast, the compactness of the B-samples is significantly higher due to intramolecular ligation. Accordingly, the tightly crosslinked C-samples adopt a mostly compact spherical conformation (close to $\alpha=0$ for solid sphere). In contrast to the clear impact of the LD on the KMH slope, the molar mass does not show significant influence on the conformation (Figure $8 \mathrm{~b}$ ).

(a)

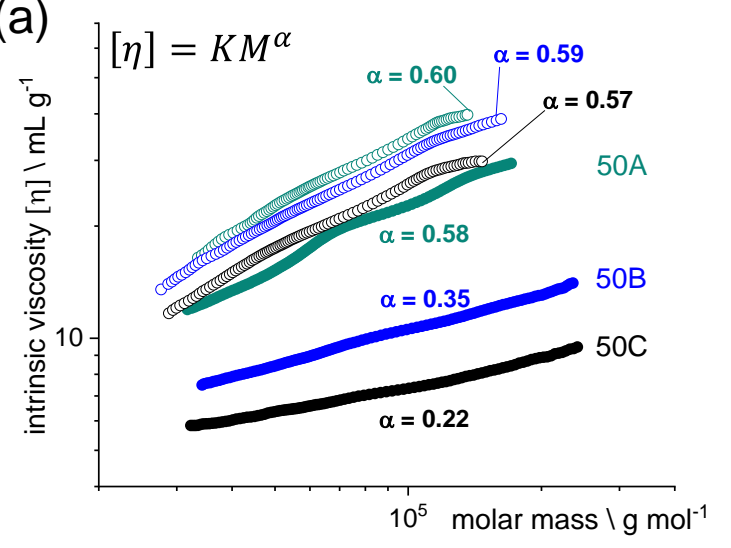

\begin{tabular}{ccc}
\hline$\alpha$ & $\begin{array}{c}\text { theta } \\
\text { solvent }\end{array}$ & $\begin{array}{c}\text { good } \\
\text { solvent }\end{array}$ \\
\hline hard sphere & 0 & 0 \\
$\begin{array}{c}\text { intramolecular } \\
\text { ligated }\end{array}$ & $0.5-0$ & 0.5 \\
$\begin{array}{c}\text { statistical coil } \\
\text { hard sphere }\end{array}$ & 0.5 & $0.6-1$ \\
\hline
\end{tabular}

(b)
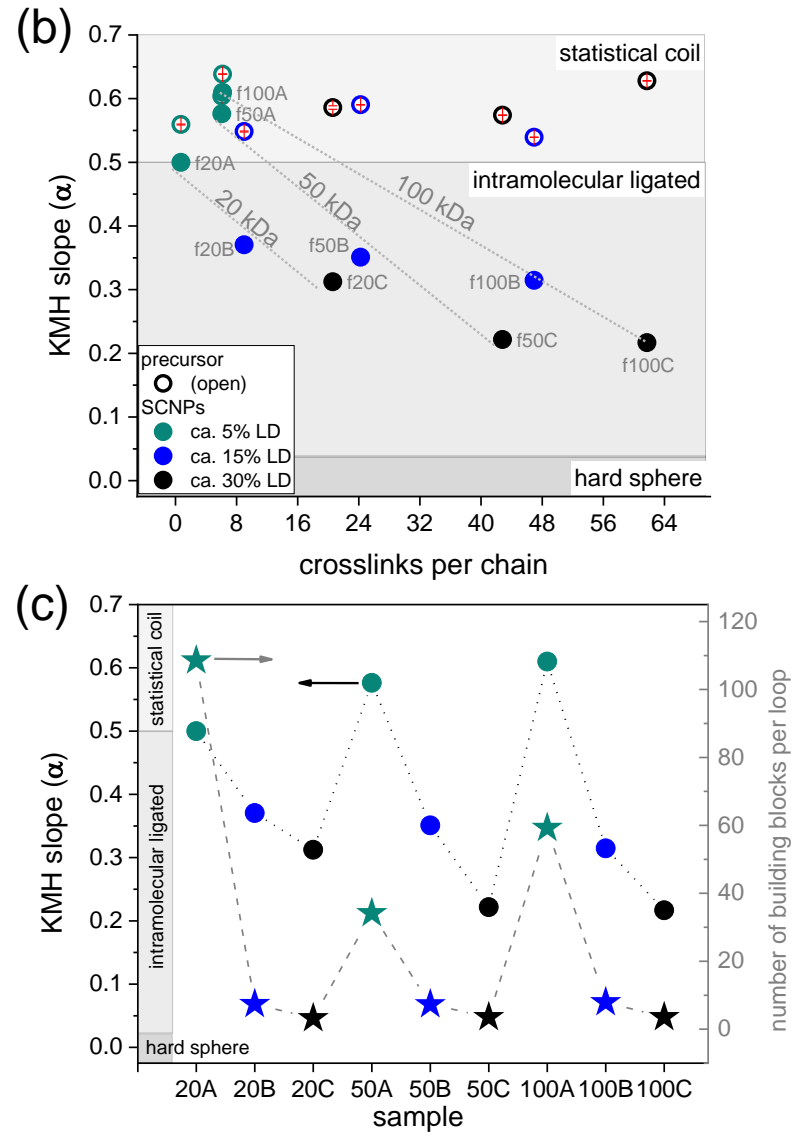

Figure 8. (a) KMH plots of the precursors (open dots) and their corresponding SCNPs (full dots) of $50 \mathrm{kDa}$ series. (b) KMH exponents of the whole copolymer library depending on the crosslinks per chain. Data for precursors (open dots) and SCNPs (full dots)are presented. Limits for statistical coil and hard sphere are indicated in the plot and in the table within the figure. (c) Correlation between loop-length (stars) calculated from the LD and polymerisation degree vs SCNP conformation according to KMH slope $\alpha$ (dots).

The knowledge about the number of crosslinks per chain provides insight into the average number of monomer units per loop, a suitable value to estimate the compactness of SCNPs. For simplicity, we can assume that the number of incorporated crosslinker units is equal to the average number of loops per chain. 
Relating the average number of loops per chain to the total number of building blocks per chain (degree of polymerisation, DP), one can estimate the number of building blocks per loop for the SCNPs, coined the loop length. Sommer and colleagues recently showed that the distances between ligation-events (considered as average loop length) follow the distribution for Gaussian chains after folding in poor solvent conditions. ${ }^{51}$ Thus, the average values of LD and polymerisation degree of the SCNPs can deliver an average of the loop length (see also SI, section 3.5.4). ${ }^{55}$ The results of the average loop length show the same trend as the results of the KMH-slopes, indicating a direct correlation of the loop length to the conventional measure of polymer conformation (Figure 8c). Interestingly, the shortest loop lengths found in the C-series correspond with approx. 4 blocks per loop to approx. $2 \mathrm{~nm}$ length (assuming $\mathrm{C}-\mathrm{C}$ bond length of $0.125 \mathrm{~nm}$ ). The shortest found block length, thus, corresponds to the average Kuhn length of $4 \mathrm{~nm}$ calculated from SANS experiments (Table 1) related to a segment length of approx. $2 \mathrm{~nm}$. This fact shows that not only the ligation density but also the segment length and flexibility of the precursor chain is a limiting factor during SCNP formation.

SEC-D4 analysis with state-of-the art detectors allows for valuable insight into the molecular conformation of the precursor library of nine precursors and their folded counterparts. While the size determination using online scattering techniques (MALS, QELS) were pushed to their limits - due to both, small sizes and low optical contrast - absolute molar mass determination and viscosity detection reliably validate the success of the folding strategy. Analysis of the viscosity and molar mass show substantial potential for size and conformation characterization, keeping in mind the applied assumptions. In order to evidence the reliability of the observed SEC-D4 data and to obtain fully comprehensive image of an SCNP architecture, a scattering technique not limited to the approximate $10 \mathrm{~nm}$ size regime or optical contrast issues is required. We have therefore employed the powerful SANS technique to sharpen our understanding of these nanoobjects.

\section{In-depth size and conformation validation via SANS}

SANS is unrivalled in analytical power for soft matter materials, as it provides the non-destructive determination of the absolute $R_{g}$ for particles down to $1 \mathrm{~nm}$ in size and additionally provides the information about the microstructure (shape and segment density) of the analyte. ${ }^{82}$ Contrary to light-scattering techniques, the contrast of SANS is given by the difference of the scattering length between a dissolved polymer and the solvent. Deuterium labelling provides decent contrast to investigate organic macromolecules and makes polymers, which have poor optical contrast, "visible". Due to the employment of THF- $\mathrm{d}_{8}$ as a solvent for SANS experiments, we have good solvent conditions for the polymer-library and excellent contrast as already demonstrated in previous studies on acrylate-based polymers ${ }^{83}$ For our SANS experiments, we selected a representative sample set of our original nine precursors and the corresponding SCNPs (samples 50A, 50B and 50C as well as 20C, 100A and 100C). Concentration dependent measurements enable the complete Zimm analysis of each sample. ${ }^{84}$ The evaluation of the scattering intensity $I(q)$ over large range of scattering vector $q$ enables information about: (i) the global scaling of the macromolecules at low $q$ values and (ii) the macromolecular segments down to the size scale of the monomers (details on the setup and theoretical background are given in SI, section 6). Figure 9a 
shows an example of a scattering curve of the 50C-pair of precursor and SCNP and the ranges of the scattering curve delivering information about size $\left(R_{g}\right)$, conformation (scaling exponent $v$ in Eq. 3 ) and flexibility (segment length or Kuhn length $l_{K}$ ) of the polymer chain (all scattering curves and their interpretation are given in SI, section 6). As the concentrations of the samples are held sufficiently below chain overlap concentration $c^{*}$ (SI, section 1), interparticle interaction is ruled out, ${ }^{85}$ as confirmed by the absence of a structure factor peak. Therefore, we can focus on the form factor $P(q)$ (SI, section 6.1) to investigate the scattering function of a single isolated polymer particle, revealing information about size and shape of the precursors and the corresponding SCNPs.

Using the Zimm analysis, the absolute $R_{g}$ and the second virial coefficients $A_{2}$ in THF- $\mathrm{d}_{8}$ were determined and are summarized in Table 1. Absolute particle sizes between 3 and $17 \mathrm{~nm}$ radius of gyration are obtained. Good agreement with the tendencies found for $R_{\eta}$ was found (Table 1, Figure 6). Furthermore, good correlation of the $R_{g}$ values with the calculated molar masses from SEC-D4 is observed: already small changes of chain length for the $50 \mathrm{kDa}$ precursor demonstrate an impact on the $R_{g}$ (samples $50 \mathrm{~A}, 50 \mathrm{~B}, 50 \mathrm{C}$ in Table 1). The extent of collapse from precursor to SCNP is in direct relation to the extent of LD and correlates with the development of the size reduction calculated from the SEC-D4. Figure 9b shows the relative volume reduction $V_{g}$ in comparison to the values calculated from the intrinsic viscosity $V_{\eta}$. The relative volume contraction is clearly dictated by the amount of crosslinks per chain. More precisely, Figure $9 \mathrm{~b}$ reveals an asymptotic increase towards a maximum relative volume contraction of approx. $80 \%$ with increasing ligation density after which a plateau is reached. These results confirm the results obtained from SEC-D4 and demonstrate that precise size-tuning of SCNPs is predominantly controlled by adjusting the precursor LD.

Having reliable information about the size reduction of the SCNPs after folding, which is confirmed by two independent analytical tools, SEC-D4 and SANS, the next logical step is to validate the reliability of the peak shift of the SEC chromatograms $\Delta V_{e}$ (Figure 5). A linear fit of the $\Delta V_{e}$ increase with increasing crosslinks per chain (Figure 9c) allows an assessment of the deviation of the normalized $\Delta V_{g}$ and $\Delta V_{\eta}$ values from this fit. Although the general tendency is similar, the plot makes immediately clear that in contrast to the well overlapping $\Delta V_{g}$ and $\Delta V_{\eta}$, their values are strongly deviating from $\Delta V_{e}$. The reason for this deviation is most likely the non-ideal entropic separation of the macromolecules, which can lead to a mixed size-exclusion / interaction mode of separation, thus, influencing the position of the peak apex of the chromatogram. Although most of the samples show linear dependence of the absolute molar mass (SEC mode) of the elution volume (see SI, section 5), some deviations from linearity can be indicated, showing that co-elution of different sizes especially in case of the SCNPs is taking place. The origin of this coelution should be investigated in depth using instruments of separation theory and advanced fractionation tools. It should be noted that for the purpose of the current study, the mixed mode of separation does not affect the calculation of the main parameters from SEC-D4 listed in Table 1 and in Tables 5-7 of the SI. However, this phenomenon of non-ideal entropic separation can be misleading in regards to the extent of folding and compaction of the SCNPs based only on the single shift of the chromatogram $\Delta V_{e}$. 

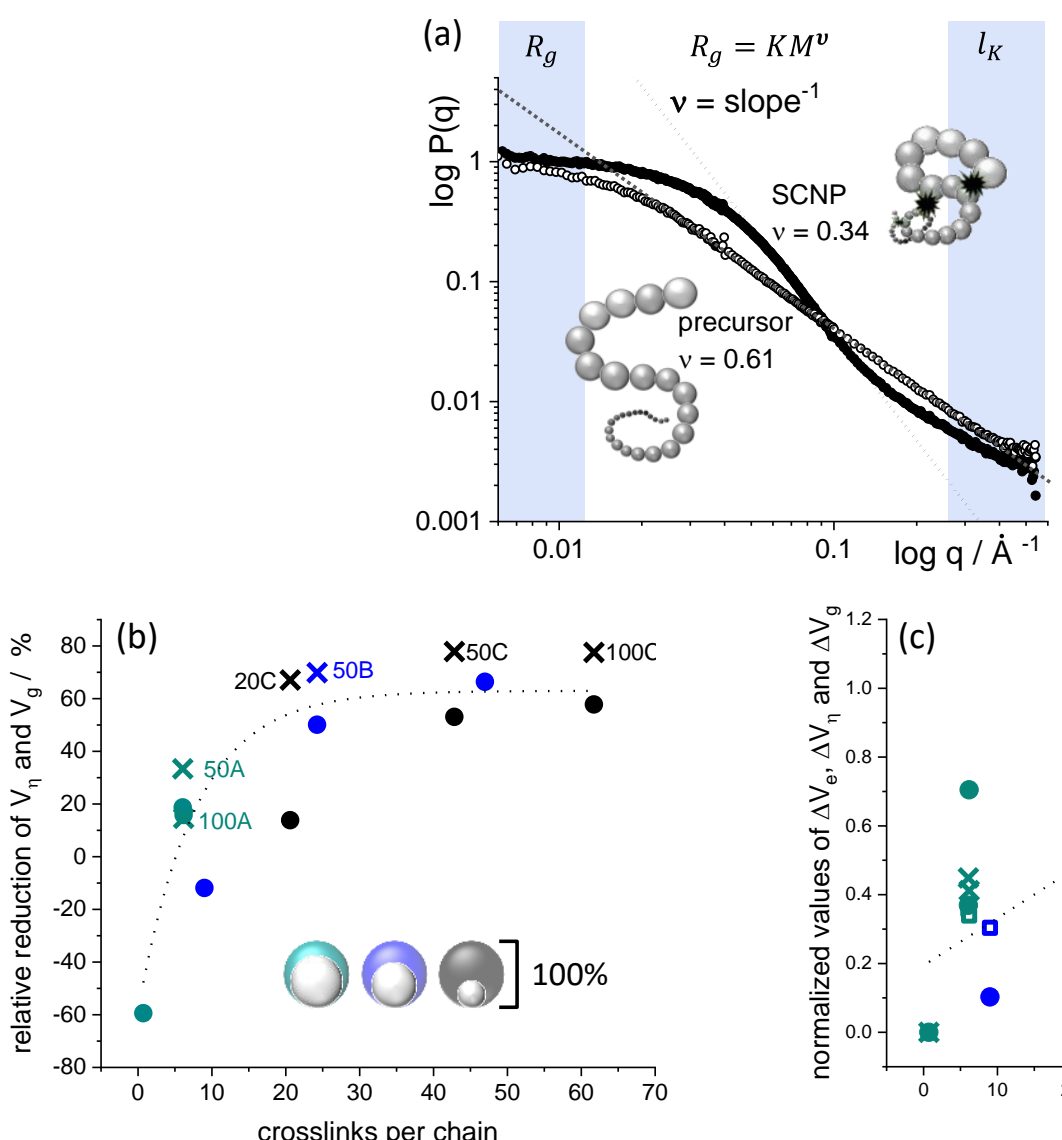

(d)
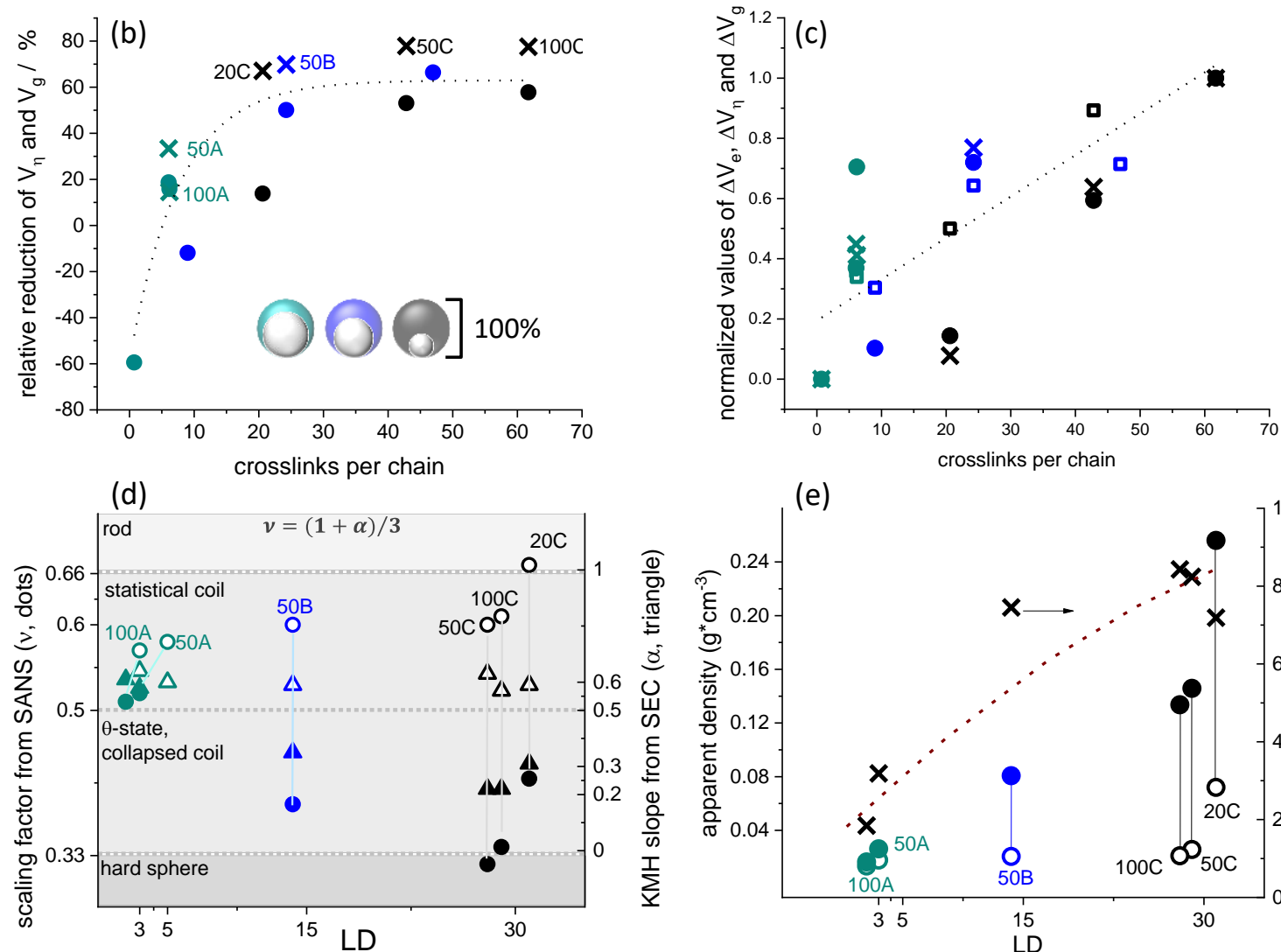

(e)
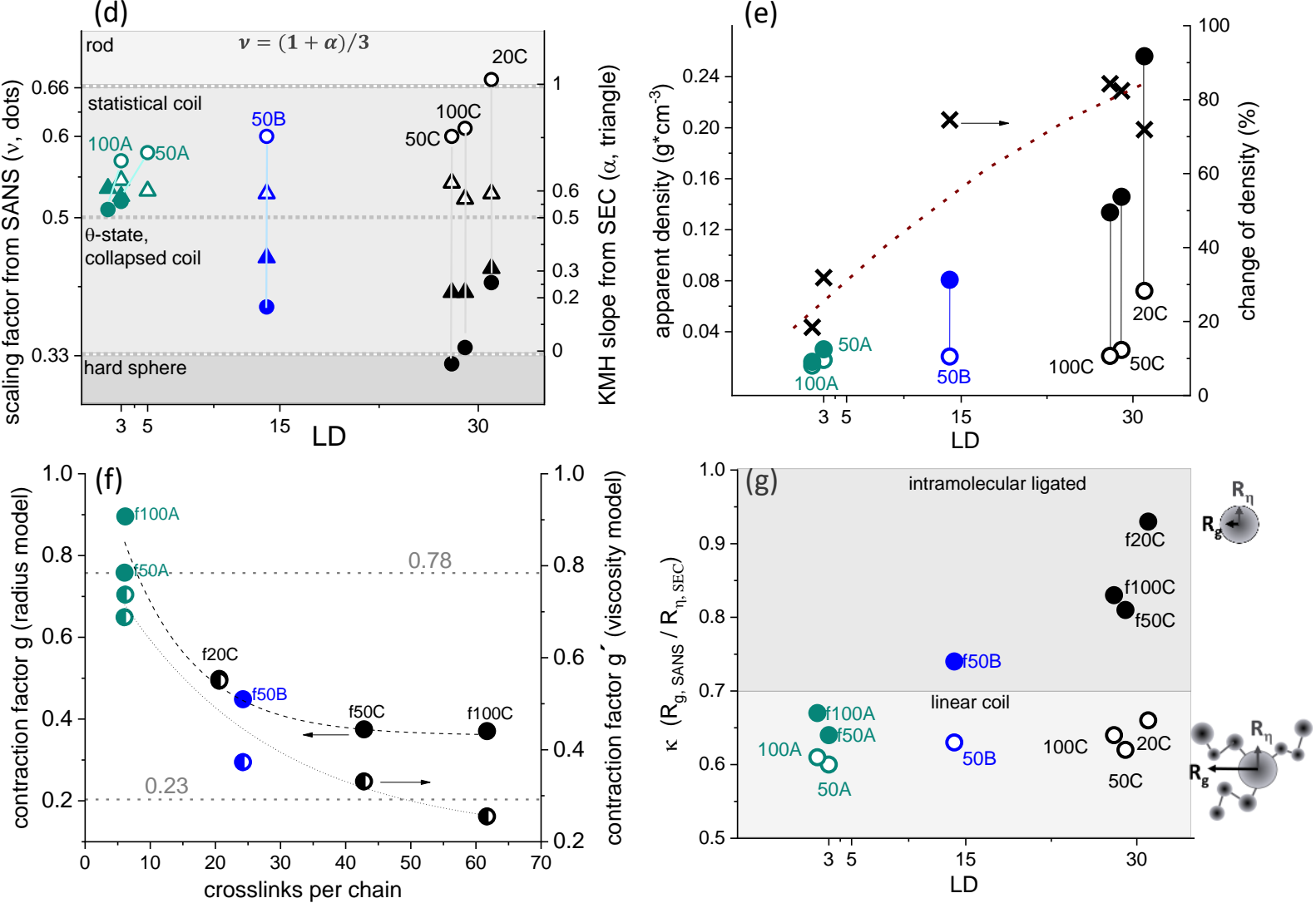

Figure 9. (a) Scattering behaviour of the precursor and the SCNP of the sample 50C with the corresponding regions of the scattering curve used for determination of $R_{g}$, scaling exponent $(v)$ and the segment length (Kuhn length, $\left.l_{K}\right)$. (b) Relative reduction of the volume $\left(\Delta V_{x} / V_{x, \text { precursor }}\right) v$ s crosslinks per chain after folding calculated from $R_{\eta}$ (dotts) and $R_{g}$ (crosses). The fit of all values should guide the eye. The scheme demonstrates the strong reduction depending on the ligation density assuming sphere shape. (c) Comparison of the normalized values of $\Delta V_{e}$ (open rectangle), $\Delta V_{\eta}$ (dotts) and $\Delta V_{g}$ (crosses) vs. crosslinks per chain. Linear fit of 


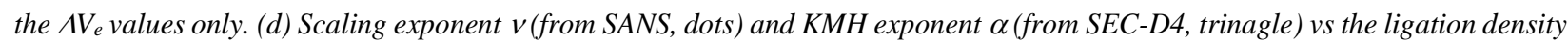
(precursors - open; SCNPs - full symbols). (e) Apparent density calculated according to Eq. 6 (precursors - open; SCNPs - full dots) and the change of the density after folding vs. $L D$ (crosses). (f) Contraction factors $g$ (full dots) and $g$ ' (half full dots) calculated according to Eqs. 7 and 8 vs crosslinks per chain. Values of $g$ found for 3-arm (0.78) and 18-arm (0.23) polystyrene stars under theta-conditions are marked in grey. ${ }^{35}(g) \kappa$-parameter, a generalised ratio between $R_{g}$ (from SANS) and $R_{\eta}$ (from SEC-D4), gives an insight into the segmental distribution and shape of the particles (see scheme) depending on the ligation density (precursors open; SCNPs - full symbols).

Further information from the Zimm analysis of the SANS results at low $q$-values delivers the second osmotic virial coefficient $A_{2}$, which is a quantitative measure of the strength of interaction between the macromolecular segments. Good solvent conditions will lead to positive $A_{2}$ values, whereas $A_{2}=0$ corresponds to unperturbed dimensions of the macromolecules under theta-conditions. The obtained second virial coefficients in THF (Table 1) are similar for most precursors indicating good solvent conditions and decreasing to theta-conditions with increasing molar mass as expected. ${ }^{35}$ Clear dependency of the $A_{2}$ from the copolymer composition is observed for the $50 \mathrm{kDa}$ samples (see Table 1). In accordance with the proposed hypothesis (Figure 7), the SCNPs show a negative $A_{2}$ close to theta-state corresponding to the adjusted and "frozen" compact conformation by folding in a poor solvent (ACN/THF).

The compact conformation of the folded SCNPs and conformation of their precursors is impressively reflected in their different scattering behaviour. Figure 9a depicts the representative chain collapse of sample 50C visible in the significantly faster decay of the scattering intensity of the SCNPs compared to the precursor. The evaluation of the negative, reciprocal slope of the scattering curves delivers information on the fractal dimension, e.g. the scaling exponent $v$, which describes the relation between the $R_{g}$ and the molar mass of the macromolecules (Eq. 3). Additional fit to model form factors (polymer excluded volume) of the scattering curves $P(q)$ was used to validate the evaluated values of the $R_{g}$ from Zimm analysis and $v$ from the slope of $P(q) .{ }^{86}$ The results from these fits show very good agreement with the manually evaluated data (see SI Table 10 and section 6.3 in the SI).

Figure $9 \mathrm{~d}$ shows that the scaling exponents of the precursor correspond to linear Gaussian chain conformation, which is defined by $v=0.6$ in a good solvent. ${ }^{87}$ Colmenero et al. recently emphasized the similarity of Gaussian chain conformation to the scaling behaviour of intrinsically disordered proteins (IDPs) defined by their biological task. ${ }^{44}$ Comparing the precursor to their corresponding SCNPs, a clear trend of increasing compaction with increasing LD is confirmed. Maximal compaction approaching spherelike conformation $(v=0.33)$ is obtained for the SCNPs of the C-series of the strongly crosslinked 50 and $100 \mathrm{kDa}$ samples, while the $20 \mathrm{kDa}$ sample show still pronounced but smaller compaction. This strong contraction is in full agreement with the slightly negative $\mathrm{A}_{2}$ values found in a good solvent (THF- $d_{8}$ ), which can only be explained by the folding strategy under carefully chosen poor solvent conditions (see Figure 7). Furthermore, the analysis of the C-series shows convincingly that the conformation changes during folding are not only depending on the frequency of the crosslinking events (LD). Having maximum reduction of the SCNP volume (at 30\% LD, Figure 9b) there is still room to control the final conformation 
from slightly compact chain conformation (20C) to sphere like (50C) to hard sphere (100C) by adjusting the absolute number of crosslinks, e.g. chain length.

The orthogonal analytical tools in this study allow for a unique comparison between the scaling exponent $v$ and the KMH exponent $\alpha$ both obtained in the same solvent conditions (THF for SEC-D4 and THF- $\mathrm{d}_{8}$ for SANS). Figure 9d further shows the $\alpha$ values relation to the $v$ values, which has its origin in the Flory mean-field theory as $v=(1+\alpha) / 3 .^{11,87,88}$ Strong agreement between both values confirms transition of the statistical precursor coils into compact, poorly drained by the solvent SCNP morphologies. The changes in $\alpha$ from SEC-D4 are, in general, less pronounced than the changes calculated by transformation of $v$ to $\alpha$ deduced by SANS. However, these deviations are within an allowable range, considering the different principle of analysis: SANS is performed in batch while SEC-D4 delivers molar mass dependent values after a separation according to size.

Increasing compaction is related with increasing density, thus, a complementary calculation of the macromolecular apparent density can validate the observed compaction. The apparent density $d_{a p p}$ is derived from the volume of gyration (evaluated via SANS characterization) and the corresponding molar mass $M_{w}$ (evaluated via SEC-D4) as follows ${ }^{87}$

$$
d_{\text {app }}=\frac{M_{w}}{V_{R_{g}}}=\frac{3}{4 \pi N_{A}} \frac{M_{w}}{R_{g}^{3}}
$$

Figure 9e shows the apparent density values as well as the change of the density after folding depending on the LD. The precursors show an average density of $d_{a p p}=0.03 \mathrm{~g} \mathrm{~cm}^{-3}$ and negligible change of this density is observed for the slightly crosslinked SCNPs (A-samples). In contrast, the medium crosslinked SCNPs show already significant increase (B-samples), and the strongly crosslinked SCNPs show the highest $d_{\text {app }}$ (C-samples). Our team earlier suggested that the minimum SCNP density is $0.1 \mathrm{~g} \mathrm{~cm}^{-3}$, despite observing a large number of SCNP systems below this density threshold. The highest found $d_{a p p}=0.25 \mathrm{~g} \mathrm{~cm}^{-3}$ for our SCNPs is far below the $1 \mathrm{~g} \mathrm{~cm}^{-3}$ expected for bulk materials, which surprisingly, was reported for some SCNPs ${ }^{80}$ It should be noted that, the swelling behaviour in a good solvent as well as bulky functional groups within the SCNP structure could strongly influence the apparent density.

The change of density upon folding increases steadily with LD (Figure 9e). The highest density is observed for the smallest SCNP sample f20C, but peculiarly, the change of density due to the folding is only changed to an extent comparable to the medium crosslinked SCNPs. In order to understand this effect more precisely, the density distribution within the macromolecule should be analysed. For this purpose, the generalised ratios $\kappa\left(R_{\eta} / R_{g}\right)$ and $\rho\left(R_{g} / R_{h}\right)$ can be used as quantitative indicators of the molecular topology of polymers in solution. ${ }^{18,89} \kappa$ indicates the mass distribution around the centre of gravity and the hydrodynamic draining of the solvent, thus giving information about the segmental density in complex macromolecules. Figure $9 \mathrm{~g}$ presents the $\kappa$ values of the samples investigated by SANS and SEC-D4. The topology of the precursors corresponds to the density-distribution of a linear coil $(\kappa=0.6)$. The increase of 
$\kappa$ is minimal for the weakly crosslinked SCNPs (A-samples), and significant for the compact SCNPs (Csamples). As a reference, the theoretical maximum compaction of solid spheres has the ratio of $\kappa=1.3 .{ }^{90}$ The SCNP sample f20C has the highest mass-centred compactness. These results are confirmed by the complementary $\rho$-parameter based on the calculation of the $R_{h}$ from SEC-D4 in combination with $R_{g}$ form SANS (SI, section 4.3.2). The observation of the $20 \mathrm{C}$ sample behaviour coincides with the phenomena observed for the viscometric radii and the apparent density of the smallest polymers. We explain this phenomena by two main reasons: ${ }^{80}$ (i) as discussed previously, shorter chains undergo predominantly short distance interactions under the poor solvent conditions due to limited number of segments able to form a loop over longer distances. Taking into account that the stiffness of the macromolecular chain is defined by the segment length, low number of segments does not allow a significant contraction in 3D sense, and the volume of the polymer coils is not significantly changed due to the folding reaction; (ii) at the same time, the mass-increase due to the incorporation of the external crosslinker contributes proportionally to the apparent density $d_{a p p}$ (Eq. 6). Visualisation of the impact of mass increase on the density upon folding for small molar mass precursors is given in the SI, section 7.4.

The reduction of macromolecular size due to conformational changes is a well-known key issue in the branching theory, where the concept of contraction factors $g$ and $g^{\prime}$ is applied. ${ }^{35,91,92}$ The contraction factors indicate the relative change of the main structure parameters used in this study, $[\eta]$ (viscosity model) and the $R_{g}$ (radius model). We define the contraction factor as the ratio of the intrinsic viscosities of the precursor and the corresponding SCNP for a given DP (Eqs. 7 and 8). ${ }^{35,88}$ The original introduction of contraction factors within the branching theory takes into account changes in [ $\eta] \quad$ or $R_{g}$ at equal molar mass of a linear polymer an the branched analogue. In our study, we translate this relationship for SCNPs contraction at similar polymerisation degrees because of the increase of the molar mass of the precursor after folding. $g$ and $g^{\prime}$ possess values between 0 and 1 , where smaller values indicate a higher contraction.

$$
\begin{aligned}
& g=\left.\frac{R_{g, \text { SCNP }}^{2}}{R_{g, \text { linear }}^{2}}\right|_{D P} \\
& g^{\prime}=\left.\frac{[\eta]_{\text {SCNP }}}{[\eta]_{\text {linear }}}\right|_{D P}
\end{aligned}
$$

Figure 9f shows that both, $g$ and $g$ ' calculated from SEC-D4 and SANS show similar tendencies. Since this is the first study in which the concept of contraction factor is applied to SCNPs, we can compare the obtained values only with reported branched polymers. As a reference, a 3-arm polystyrene star under thetaconditions shows weak contraction with $\mathrm{g}=0.78$ while an 18 -arms polystyrene strong contraction with $g$ $=0.23$ is reported.$^{35}$ It should be noted that the nature of the contraction factor is an independent measure and neither the architecture, nor chemical structure and solvent conditions need to be considered. The experimental data in Figure 9f demonstrate the direct correlation of the contraction factors to the crosslinks per chain revealing the minor impact of the molar mass, but strong impact of the crosslinking events. The samples with comparable LD (for example the C-samples, black) show only slightly different contraction factors, indicating that the extent of contraction increases slightly with the length of the precursor chain. As 
seen by several previous parameters, the smallest polymer (sample 20C) shows smaller contraction in comparison to larger polymers of the same LD, which supports our hypothesis of limited conformational freedom during folding for such small polymers. In conclusion, for quantification of the relative compaction of the SCNPs, contraction factors show better suitability than the apparent density $d_{a p p}$. Further details on the relation ${ }^{87,88}$ between the $g$ and $g$ ' are given in the SI, section 7.3.

The SANS scattering curves of our library contain much more information than only the structural parameters discussed above. The extraction of this information can be performed by analysis of the scattering intensity $P(q)$ according to Kratky or Casassa ${ }^{84,93,94}$ Kratky and Casassa plots can qualitatively assess the degree of folding and are suitable for determination of the flexibility of linear chains using the high $q$-regime (see Figure 9a). This analysis is usually applied to concentration dependent scattering curves, which leads to simplified data analysis. Although, Hawker et al. demonstrated in early SCNP research, that no remarkable differences of Kratky plots are given for different concentrations for slightly branched polystyrene-based SCNPs, ${ }^{40}$ we plot the form factor $P(q)$ corrected for angle and concentration dependency for sake of accuracy as obtained by our Zimm analysis.

Kratky plots of the dimension $q^{2} P(q)$ vs. $q$ facilitate to decipher the segment density, as a plateau appears for polymers of a Gaussian coil conformation ( $q^{-2}$-slope). In contrast, globular structures show a bell-shaped peak (Figure 10c). ${ }^{95,96}$ Kratky plots have been frequently applied to reveal the folding state of proteins, by referring the area of the bell above the plateau to the degree of folding. ${ }^{95}$ This calculation approach can therefore, semi-qualitatively assess the degree of intramolecular ligation, as the position of the local maximum refers to the linear mass fractions of the particles. ${ }^{93,96}$ Kratky plots have already been applied to experimental SANS data of SCNPs to evaluate the degree of compaction, for example by comparison to Kratky plots of form factors for globular particles. ${ }^{36-40}$ Nevertheless, we exercise caution when directly comparing Kratky plots of completely different structures, as performed for the scattering form factors of SCNPs and intrinsically disordered proteins to that of compact globular proteins. ${ }^{36}$ Instead, we compare the Kratky plots within a chemically similar system (e.g. precursor and the correlated SCNPs, or SCNPs of the same building blocks) to elucidate the density and sphericity of the polymers of our polymer library (Figure 10). For all precursors, a plateau in a Kratky plot is obtained, resembling a Gaussian coil conformation (see SI, section 6). Increasing plateau height is, amongst other factors, caused by the different particle sizes (molar mass), which can be shown in a dimensionless Kratky plot $\left(q R_{g}\right)^{2} P(q)$ vs. $q R_{g}$, see SI, section 6.1.2). Dimensionless Kratky plots have been, for example, applied to synthetic SCNPs, intrinsically disordered proteins and globular proteins by Pomposo et al. ${ }^{36}$ In Figure 10a the $50 \mathrm{kDa}$ series of the precursors are shown. Figure 10b shows the Kratky plots of the 50kDa SCNPs (all plots available in SI, section 6). In contrast to the less crosslinked SCNPs (A-samples), the strongly crosslinked SCNPs (B- and C-samples) show a pronounced Gaussian distribution in the Kratky plot indicating globular particles as also reported for globular proteins. ${ }^{95}$ These local maxima of the Kratky plot indicate an increased segment density. The position of the apex of the peak (in $q$-dimension) is related to the size of the mass-fractals and resembles the trends observed for $R_{g}$ of the SCNPs (Table 1). Contrary to the classical Kratky plots of spheres, a 
plateau is still present for the compact B- and C-series of the SCNPs at high $q$-values. This observation indicates the coexistence of multiple domains composed of dense-fractions and linear chain conformations also observed for proteins, ${ }^{97}$ leading to the assumption that the macromolecule does not possess homogenous segment distribution, but linear segments and dense segments jointly exist. ${ }^{93,96}$ The denser segmented regions in the SCNP structures can be described as cross-linking crowded regions however, this assumption needs further elucidation using theoretical consideration in future studies.
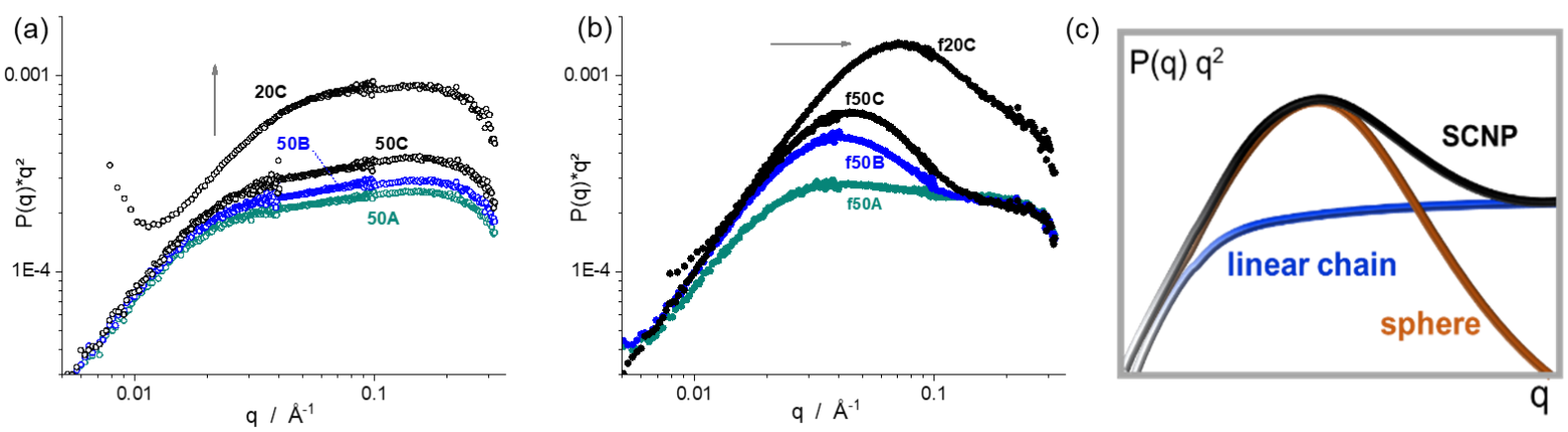

Figure 10. Kratky plots of $50 \mathrm{kDa}(A, B, C$-samples) and 20kDa (C-sample): (a) linear precursor polymers and (b) SCNP polymers. The arrows indicate the shift of the maxima in direction of decreasing molar mass (a) and on the mass fraction in direction of decreasing size (b). (c) Schematic Kratky presentation of typical tendencies expected and found for linear chain, hard sphere and SCNPs.

Closer look at the 20C SCNPs (Figure 10b) shows that such a plateau is less pronounced for this small molar mass sample. This observation, analysed in the context of the calculated high apparent density and high segment density found for this particular sample (Figure 9e and 9g), confirms low amounts of linear segments but rather dense structure with small dimensions. These findings support the above discussed hypothesis of short distance interactions responsible for crosslinking at low degrees of polymerisation, which cannot lead to joined linear and dense, globular segments in one SCNP as in the case of the 50 and $100 \mathrm{kDa}$ samples.

Similar to the Kratky plots, the Casassa plots of the SCNPs show a significantly increased maximum at low $q$-ranges (SI, section 6), which is typical for dense coil-structures. We apply this plot to deduce semiquantitative information about the coil behaviour itself, as the height of the maximum increases with decreasing deformability. The transition (denoted with $q *$ in SI, section 6.1.3) from coil-like (low $q$-range) to rod-like behaviour (high $q$-range) enables the calculation of the segment length. Another way to calculate the segment length is using a fit with model form factors as described in the SI, section 6.3.1. leading to precise analysis of the particle's features. The form factor model flexible cylinder provides the ability to determine the Kuhn length $l_{K}$, based on simulations of a discrete representation of a worm-like chain model of Kratky and Porod and normalized by the volume of a flexible cylinder. ${ }^{98}$ For this purpose the SasView ${ }^{86}$ application was used, as described in the SI, section 6. Very good fit quality was achieved for all precursor samples and assures high reliability of the obtained data. The obtained Kuhn lengths of the precursors between 3.2 and $4.2 \mathrm{~nm}$ are slightly lower than the approx. $4.6 \mathrm{~nm}$ found for poly $\left({ }^{t} \mathrm{Bu}\right.$ acrylate) ${ }^{83}$ The shortest $l_{K}$, thus, the most flexible chain, is found for the precursor 20C, whereas sample 50C shows the 
longest $l_{K}$-although both precursors exhibit a comparable backbone composition. Generally, the A-samples show better flexibility, thus, the low crosslinking turnover of the low functionalized precursor is not caused by steric hindrance of potentially stiff backbone features, but is solely caused by statistically reduced intramolecular ligation events. Focusing on the $50 \mathrm{kDa}$ samples, a clear increase of $l_{K}$ is indicated, which correlates well with the decreasing solubility in the order A > B > C. From these reasons, we assume maximal flexibility of the polymer chain of A-precursors. The solvent quality and the chain stiffness, both in means of the degree of rotational freedom, are of paramount importance for the efficacy of the folding reaction. ${ }^{99,} 100$ As the bending stiffness of the linear precursor determines the probability of intramolecular interaction in space, and therefore influences the morphology of the SCNPs, we conclude negligible influence of changing backbone composition or stiffness on the SCNP morphology.

\section{Conclusions}

Regioselective para-fluoro thiol cross-linking allows for the precise design of a polymer library and its systematic folding into SCNPs defined by predetermined numbers of linkage points and molar masses. Thus, two general properties of the precursor structure are investigated regarding their impacts on the SCNPs properties, making findings applicable to SCNPs regardless of the employed chemical strategy of the folding reaction.

Extensive ${ }^{1} \mathrm{H}$ NMR spectroscopy, ${ }^{19} \mathrm{~F}$ NMR spectroscopy, SEC-D4 and SANS provide unique insight into the parameters limiting the formation of SCNPs. We combine these orthogonal instruments of advanced polymer analysis to obtain a multidimensional picture of the resulting architectures - from the chemical structure to the segment distribution to the global conformation.

The turnover of the crosslinking reaction and resulting size reduction of the formed SCNPs relative to the linear precursors directly correlates with two main parameters: the ligation density and the conformation of the linear precursor under the conditions of the folding reaction.

Based on molar masses from 20 to $100 \mathrm{kDa}$ and ligation densities from 3 to $30 \%$ of the precursors, we estimate $(i)$ a lower limit of $5 \%$ ligation density for effective crosslinking with minimum size reduction during SCNP formation and (ii) a higher limit of 30\% ligation density leading to maximum achievable size reduction of $80 \%$. These limits are additionally influenced by the molar mass of the precursor. For molar masses of $20 \mathrm{kDa}, 80 \%$ volume contraction is not achievable due to lack of long-distance interactions as a result of low number of segments, which enable limited variation of the conformation even under poor solvent conditions.

In the molar mass range between 20 and $100 \mathrm{kDa}$, an effective control of the size reduction can be observed mainly depending on the absolute number of crosslinks per chain in the range between 5 and 40. These ranges are entirely independent on the chain length for molar masses above $20 \mathrm{kDa}$. For more than 40 crosslinks at sufficiently high molar masses, no further increase of the compaction can be indicated. 
These results are validated by the systematic combination of complementary scaling approaches using key parameters including molar mass, second virial coefficient, segment length, intrinsic viscosity, gyration, hydrodynamic and viscosity radii and apparent density. As an alternative to the apparent density, we introduce the contraction ratios $g$ and $g^{\prime}$ as simple yet meaningful tools to compare SCNPs properties regardless of the synthetic strategy. Calculations of the scaling exponent as well as the complementary Kuhn-Mark-Houwink exponent unlock the highest achievable density for the 50 and 100kDa polymers leading to a hard sphere shape. However, the segment density based on generalized ratios of different radii and detailed Kratky and Casassa analysis of the SANS scattering behaviour unravel joint dense globular and linear segments within these SCNP structures.

These fundamental insights into the parameters ruling the formation of SCNPs enable the precise assessment of the commonly used shift of the elution volume using SEC in comparison with multidetector SEC-D4 and profound validation of these results by SANS. While SEC-D4 has its limits in terms of the use of optical detectors due to very small SCNP sizes and low refractive index increment, it is a reliable technique for SCNP analysis, when viscosity detection is used. The accuracy of these experiments is, however, essentially dependent on precise absolute molar mass analysis using MALS detector and the careful determination of the refractive index increment.

The obtained results from SANS and SEC-D4 demonstrate that the shift of the elution volume, even if mainly entropic separation (according to particle size) is operational, cannot be used as a reliable detection of size reduction due to SCNP folding. The reasons of this limitation can be found in the changes of the chemical composition after folding as well as in the very complex conformation of the SCNP structures. Further studies on the separation mechanisms in SEC should shed light on this question.

When exploring approaches for size tuned artificial nanocontainers, SCNPs represent an ideal avenue between synthetically demanding dendrimers and architectural delicate macro-complexes such as micelles or polymersomes. Exploiting the chemistry available for SCNP construction, we uncover general guidelines for the tailored design of SCNPs with regard to molar mass and number of linkable units for the precursor material. The introduced alternative parameters such as $g, g$, and $\kappa$ show better applicability than conventional ones such as the apparent density and size that are of generalized use for encapsulation, retention behaviour or for catalytic activities, and bring SCNPs applications within reach. 


\section{Acknowledgements}

C.B.-K. acknowledges the Australian Research Council (ARC) for funding in the context of a Laureate Fellowship as well as the Queensland University of Technology (QUT) for continued key support. The current work further benefited from the use of the SasView application, originally developed under NSF award DMR-0520547. SasView contains code developed with funding from the European Union's Horizon 2020 research and innovation programme under the SINE2020 project, grant agreement No 654000. B. T. T. gratefully thanks Dr S Walden of QUT for helpful conversations in the preparation of this manuscript. SANS experimental data were generated under the ILL project doi:10.5291/ILL-DATA.9-11-1908.

\section{References}

(1) Mazmanian, K.; Sargsyan, K.; Lim, C. How the Local Environment of Functional Sites Regulates Protein Function. J. Am. Chem. Soc. 2020, 142 (22), 9861-9871.

(2) Engelke, J.; Brandt, J.; Barner-Kowollik, C.; Lederer, A. Strengths and Limitations of Size Exclusion Chromatography for Investigating Single Chain Folding - Current Status and Future Perspectives. Polym. Chem. 2019, 10 (25), 3410-3425.

(3) Lyon, C. K.; Prasher, A.; Hanlon, A. M.; Tuten, B. T.; Tooley, C. A.; Frank, P. G.; Berda, E. B. A Brief User's Guide to Single-Chain Nanoparticles. Polym. Chem. 2015, 6 (2), 181-197.

(4) Moreno, A. J.; Lo Verso, F.; Arbe, A.; Pomposo, J. A.; Colmenero, J. Concentrated Solutions of Single-Chain Nanoparticles: A Simple Model for Intrinsically Disordered Proteins under Crowding Conditions. J. Phys. Chem. Lett. 2016, 7 (5), 838-844.

(5) Stals, P. J. M.; Li, Y.; Burdyńska, J.; Nicolaÿ, R.; Nese, A.; Palmans, A. R. A.; Meijer, E. W.; Matyjaszewski, K.; Sheiko, S. S. How Far Can We Push Polymer Architectures? J. Am. Chem. Soc. 2013, 135 (31), 11421-11424.

(6) Perez-Baena, I.; Asenjo-Sanz, I.; Arbe, A.; Moreno, A. J.; Lo Verso, F.; Colmenero, J.; Pomposo, J. A. Efficient Route to Compact Single-Chain Nanoparticles: Photoactivated Synthesis via ThiolYne Coupling Reaction. Macromolecules 2014, 47 (23), 8270-8280.

(7) Foster, E. J.; Berda, E. B.; Meijer, E. W. Tuning the Size of Supramolecular Single-Chain Polymer Nanoparticles. J. Polym. Sci. Part A Polym. Chem. 2011, 49 (1), 118-126.

(8) Pomposo, J. A.; Rubio-Cervilla, J.; Moreno, A. J.; Lo Verso, F.; Bacova, P.; Arbe, A.; Colmenero, J. Folding Single Chains to Single-Chain Nanoparticles via Reversible Interactions: What Size Reduction Can One Expect? Macromolecules 2017, 50 (4), 1732-1739.

(9) Huurne, G. M.; Palmans, A. R. A.; Meijer, E. W. Supramolecular Single-Chain Polymeric Nanoparticles. CCS Chem. 2019, 64-82.

(10) Rothfuss, H.; Knöfel, N. D.; Roesky, P. W.; Barner-Kowollik, C. Single-Chain Nanoparticles as Catalytic Nanoreactors. J. Am. Chem. Soc. 2018, 140 (18), 5875-5881.

(11) Kröger, A. P. P.; Paulusse, J. M. J. Single-Chain Polymer Nanoparticles in Controlled Drug Delivery and Targeted Imaging. J. Control. Release 2018, 286 (June), 326-347.

(12) Palmans, A. R. A. Single-Chain Polymeric Nanoparticles: Toward in Vivo Imaging and Catalysis in Complex Media. In Self-Assembling Biomaterials: Molecular Design, Characterization and Application in Biology and Medicine; Elsevier, 2018; pp 563-583.

(13) Willenbacher, J.; Altintas, O.; Trouillet, V.; Knöfel, N.; Monteiro, M. J.; Roesky, P. W.; BarnerKowollik, C. Pd-Complex Driven Formation of Single-Chain Nanoparticles. Polym. Chem. 2015, 6 (24), 4358-4365. 
(14) Fischer, T. S.; Schulze-Suenninghausen, D.; Luy, B.; Altintas, O.; Barner-Kowollik, C. Stepwise Unfolding of Single-Chain Nanoparticles by Chemically Triggered Gates. Angew. Chemie - Int. Ed. 2016, 55 (37), 11276-11280.

(15) Perez-Baena, I.; Barroso-Bujans, F.; Gasser, U.; Arbe, A.; Moreno, A. J.; Colmenero, J.; Pomposo, J. A. Endowing Single-Chain Polymer Nanoparticles with Enzyme-Mimetic Activity. ACS Macro Lett. 2013, 2 (9), 775-779.

(16) Frisch, H.; Tuten, B. T.; Barner-Kowollik, C. Macromolecular Superstructures: A Future Beyond Single Chain Nanoparticles. Isr. J. Chem. 2020, 60 (1-2), 86-99.

(17) Frank, P.; Prasher, A.; Tuten, B.; Chao, D.; Berda, E. Characterization of Single-Chain Polymer Folding Using Size Exclusion Chromatography with Multiple Modes of Detection. Appl. Petrochemical Res. 2015, 5 (1), 9-17.

(18) Plüschke, L.; Mundil, R.; Sokolohorskyj, A.; Merna, J.; Sommer, J.-U.; Lederer, A. High Temperature Quadruple-Detector Size Exclusion Chromatography for Topological Characterization of Polyethylene. Anal. Chem. 2018, 90 (10), 6178-6186.

(19) Tooley, C. A.; Pazicni, S.; Berda, E. B. Toward a Tunable Synthetic [FeFe] Hydrogenase Mimic: Single-Chain Nanoparticles Functionalized with a Single Diiron Cluster. Polym. Chem. 2015, 6 (44), 7646-7651.

(20) Tuten, B. T.; Chao, D.; Lyon, K.; Berda, E. B. Single-Chain Polymer Nanoparticles via Reversible Disulfides Bridges. Polym. Chem. 2012, 3, 3068-3071.

(21) Frank, P. G.; Tuten, B. T.; Prasher, A.; Chao, D.; Berda, E. B. Intra-Chain Photodimerization of Pendant Anthracene Units as an Efficient Route to Single-Chain Nanoparticle Fabrication. Macromol. Rapid Commun. 2014, 35 (2), 249-253.

(22) Lyon, C. K.; Hill, E. O.; Berda, E. B. Zipping Polymers into Nanoparticles via Intrachain Alternating Radical Copolymerization. Macromol. Chem. Phys. 2016, 217 (3), 501-508.

(23) Chen, R.; Dickinson, J. G.; Rodriguez, K. J.; Hanlon, A. M.; Berda, E. B.; Willis, C.; Cashman, M. Scalable Synthesis of Single-Chain Nanoparticles under Mild Conditions. Macromolecules 2017, 50 (7), 2996-3003.

(24) Basasoro, S.; Gonzalez-Burgos, M.; Moreno, A. J.; Verso, F. Lo; Arbe, A.; Colmenero, J.; Pomposo, J. A. A Solvent-Based Strategy for Tuning the Internal Structure of Metallo-Folded Single-Chain Nanoparticles. Macromol. Rapid Commun. 2016, 37 (13), 1060-1065.

(25) Prasher, A.; Loynd, C. M.; Tuten, B. T.; Frank, P. G.; Chao, D.; Berda, E. B. Efficient Fabrication of Polymer Nanoparticles via Sonogashira Cross-Linking of Linear Polymers in Dilute Solution. $J$. Polym. Sci. Part A Polym. Chem. 2016, 54 (1), 209-217.

(26) Nitsche, T.; Steinkoenig, J.; De Bruycker, K.; Bloesser, F. R.; Blanksby, S. J.; Blinco, J. P.; BarnerKowollik, C. Mapping the Compaction of Discrete Polymer Chains by Size Exclusion Chromatography Coupled to High-Resolution Mass Spectrometry. Macromolecules 2019, 52 (6), 2597-2606.

(27) Hosono, N.; Kushner, A. M.; Chung, J.; Palmans, A. R. A.; Guan, Z.; Meijer, E. W. Forced Unfolding of Single-Chain Polymeric Nanoparticles. J. Am. Chem. Soc. 2015, 137 (21), 6880-6888.

(28) Willenbacher, J.; Wuest, K. N. R.; Mueller, J. O.; Kaupp, M.; Wagenknecht, H.-A.; BarnerKowollik, C. Photochemical Design of Functional Fluorescent Single-Chain Nanoparticles. ACS Macro Lett. 2014, 3 (6), 574-579.

(29) Zhang, J.; Gody, G.; Hartlieb, M.; Catrouillet, S.; Moffat, J.; Perrier, S. Synthesis of SequenceControlled Multiblock Single Chain Nanoparticles by a Stepwise Folding of Chain Extension by Folding Process. Macromolecules 2016, 49 (23), 8933-8942.

(30) Hosono, N.; Gillissen, M. A. J.; Li, Y.; Sheiko, S. S.; Palmans, A. R. A.; Meijer, E. W. Orthogonal Self-Assembly in Folding Block Copolymers. J. Am. Chem. Soc. 2013, 135 (1), 501-510.

(31) Cui, Z.; Cao, H.; Ding, Y.; Gao, P.; Lu, X.; Cai, Y. Compartmentalization of an ABC Triblock Copolymer Single-Chain Nanoparticle via Coordination-Driven Orthogonal Self-Assembly. Polym. 
Chem. 2017, 8 (24), 3755-3763.

(32) Berda, E. B.; Foster, E. J.; Meijer, E. W. Toward Controlling Folding in Synthetic Polymers: Fabricating and Characterizing Supramolecular Single-Chain Nanoparticles. Macromolecules 2010, 43 (3), 1430-1437.

(33) Foster, E. J.; Berda, E. B.; Meijer, E. W. Metastable Supramolecular Polymer Nanoparticles via Intramolecular Collapse of Single Polymer Chains. J. Am. Chem. Soc. 2009, 131 (20), 6964-6966.

(34) Wen, J.; Zhang, J.; Zhang, Y.; Yang, Y.; Zhao, H. Controlled Self-Assembly of Amphiphilic Monotailed Single-Chain Nanoparticles. Polym. Chem. 2014, 5 (13), 4032.

(35) Burchard, W. Solution Properties of Branched Macromolecules; Springer Berlin Heidelberg: Berlin, Heidelberg, 1999; Vol. 143.

(36) Pomposo, J. A.; Perez-Baena, I.; Lo Verso, F.; Moreno, A. J.; Arbe, A.; Colmenero, J. How Far Are Single-Chain Polymer Nanoparticles in Solution from the Globular State? ACS Macro Lett. 2014, 3 (8), 767-772.

(37) Sanchez-Sanchez, A.; Akbari, S.; Etxeberria, A.; Arbe, A.; Gasser, U.; Moreno, A. J.; Colmenero, J.; Pomposo, J. A. "Michael” Nanocarriers Mimicking Transient-Binding Disordered Proteins. ACS Macro Lett. 2013, 2 (6), 491-495.

(38) Arbe, A.; Pomposo, J. A.; Asenjo-Sanz, I.; Bhowmik, D.; Ivanova, O.; Kohlbrecher, J.; Colmenero, J. Single Chain Dynamic Structure Factor of Linear Polymers in an All-Polymer Nano-Composite. Macromolecules 2016, 49 (6), 2354-2364.

(39) Hansell, C. F.; Lu, A.; Patterson, J. P.; O’Reilly, R. K. Exploiting the Tetrazine-Norbornene Reaction for Single Polymer Chain Collapse. Nanoscale 2014, 6 (8), 4102-4107.

(40) Mackay, M. E.; Dao, T. T.; Tuteja, A.; Ho, D. L.; Van Horn, B.; Kim, H. C.; Hawker, C. J. Nanoscale Effects Leading to Non-Einstein-like Decrease in Viscosity. Nat. Mater. 2003, 2 (11), 762-766.

(41) De-La-Cuesta, J.; González, E.; Moreno, A. J.; Arbe, A.; Colmenero, J.; Pomposo, J. A. Size of Elastic Single-Chain Nanoparticles in Solution and on Surfaces. Macromolecules 2017, 50 (16), 6323-6331.

(42) Arbe, A.; Pomposo, J. A.; Moreno, A. J.; LoVerso, F.; González-Burgos, M.; Asenjo-Sanz, I.; Iturrospe, A.; Radulescu, A.; Ivanova, O.; Colmenero, J. Structure and Dynamics of Single-Chain Nano-Particles in Solution. Polym. (United Kingdom) 2016, 105, 532-544.

(43) Yoshikawa, K.; Yoshinaga, N. Novel Scenario of the Folding Transition of a Single Chain. J. Phys. Condens. Matter 2005, 17 (31), 2817.

(44) González-Burgos, M.; Arbe, A.; Moreno, A. J.; Pomposo, J. A.; Radulescu, A.; Colmenero, J. Crowding the Environment of Single-Chain Nanoparticles: A Combined Study by SANS and Simulations. Macromolecules 2018, 51 (4), 1573-1585.

(45) Rubio-Cervilla, J.; Malo de Molina, P.; Robles-Hernández, B.; Arbe, A.; Moreno, A. J.; Alegría, A.; Colmenero, J.; Pomposo, J. A. Facile Access to Completely Deuterated Single-Chain Nanoparticles Enabled by Intramolecular Azide Photodecomposition. Macromol. Rapid Commun. 2019, 40 (9).

(46) Moreno, A. J.; Lo Verso, F.; Sanchez-Sanchez, A.; Arbe, A.; Colmenero, J.; Pomposo, J. A. Advantages of Orthogonal Folding of Single Polymer Chains to Soft Nanoparticles. Macromolecules 2013, 46 (24), 9748-9759.

(47) Danilov, D.; Barner-Kowollik, C.; Wenzel, W. Modelling of Reversible Single Chain Polymer SelfAssembly: From the Polymer towards the Protein Limit. Chem. Commun. Chem. Commun 2015, 6002 (51), 6002-6005.

(48) Liu, J. W.; Mackay, M. E.; Duxbury, P. M. Molecular Dynamics Simulation of Intramolecular Cross-Linking of BCB/Styrene Copolymers. Macromolecules 2009, 42 (21), 8534-8542.

(49) Lo Verso, F.; Pomposo, J. A.; Colmenero, J.; Moreno, A. J. Tunable Slow Dynamics in a New Class of Soft Colloids. Soft Matter 2016, 12 (44), 9039-9046.

(50) Gartner, T. E.; Jayaraman, A. Modeling and Simulations of Polymers: A Roadmap. Macromolecules 
2019, 52 (3), 755-786.

(51) Rabbel, H.; Breier, P.; Sommer, J. U. Swelling Behavior of Single-Chain Polymer Nanoparticles: Theory and Simulation. Macromolecules 2017, 50 (18), 7410-7418.

(52) Guo, Y.; Werner, M.; Li, W.; Sommer, J.-U.; Baulin, V. A. Shape-Adaptive Single-Chain Nanoparticles Interacting with Lipid Membranes. Macromolecules 2019, 52 (24), 9578-9584.

(53) Noy, J.; Friedrich, A.; Batten, K.; Bhebhe, M. N.; Busatto, N.; Batchelor, R. R.; Kristanti, A.; Pei, Y.; Roth, P. J. Para-Fluoro Postpolymerization Chemistry of Poly(Pentafluorobenzyl Methacrylate): Modification with Amines, Thiols, and Carbonylthiolates. Macromolecules 2017, 50 (18), 7028 7040 .

(54) Cavalli, F.; Bloesser, F. R.; Barner-Kowollik, C.; Barner, L. Self-Propagated Para-Fluoro-Thiol Reaction. Chem. - A Eur. J. 2019, 25 (43), 10049-10053.

(55) Altintas, O.; Barner-Kowollik, C. Single-Chain Folding of Synthetic Polymers: A Critical Update. Macromol. Rapid Commun. 2016, 37 (1), 29-46.

(56) Daikos, O.; Naumov, S.; Knolle, W.; Heymann, K.; Scherzer, T. Peculiarities of the PhotoinitiatorFree Photopolymerization of Pentabrominated and Pentafluorinated Aromatic Acrylates and Methacrylates. Phys. Chem. Chem. Phys. 2016, 18 (47), 32369-32377.

(57) Dietrich, M.; Glassner, M.; Gruendling, T.; Schmid, C.; Falkenhagen, J.; Barner-Kowollik, C. Facile Conversion of RAFT Polymers into Hydroxyl Functional Polymers: A Detailed Investigation of Variable Monomer and RAFT Agent Combinations. Polym. Chem. 2010, 1 (5), 634.

(58) Sargent, M. V; Dean, F. M. 3.11 - Furans and Their Benzo Derivatives: (Ii) Reactivity. In Comprehensive Heterocyclic Chemistry; Katritzky, A. R., Rees, C. W., Eds.; Pergamon: Oxford, 1984; pp 599-656.

(59) Chong, Y. K.; Moad, G.; Rizzardo, E.; Thang, S. H. Thiocarbonylthio End Group Removal from RAFT-Synthesized Polymers by Radical-Induced Reduction. Macromolecules 2007, 40 (13), 44464455 .

(60) Moad, G.; Rizzardo, E.; Thang, S. H. End-Functional Polymers, Thiocarbonylthio Group Removal/Transformation and Reversible Addition-Fragmentation-Chain Transfer (RAFT) Polymerization. Polym. Int. 2011, 60 (1), 9-25.

(61) Meiser, W. Investigation of the Kinetics and Mechanism of RAFT Polymerization via EPR Spectroscopy, Georg-August-Universität Göttingen, 2012.

(62) Chen, J.; Wang, J.; Bai, Y.; Li, K.; Garcia, E. S.; Ferguson, A. L.; Zimmerman, S. C. Enzyme-like Click Catalysis by a Copper-Containing Single-Chain Nanoparticle. J. Am. Chem. Soc. 2018, 140 (42), 13695-13702.

(63) Cavalli, F.; De Keer, L.; Huber, B.; Van Steenberge, P. H. M.; D’Hooge, D. R.; Barner, L. A Kinetic Study on the: Para -Fluoro-Thiol Reaction in View of Its Use in Materials Design. Polym. Chem. 2019, 10 (22), 2781-2791.

(64) Nair, D. P.; Podgorski, M.; Chatani, S.; Gong, T.; Xi, W.; Fenoli, C. R.; Bowman, C. N. The ThiolMichael Addition Click Reaction: A Powerful and Widely Used Tool in Materials Chemistry. Chem. Mater. 2014, 26 (1), 724-744.

(65) Pauloehrl, T.; Delaittre, G.; Bastmeyer, M.; Barner-Kowollik, C. Ambient Temperature Polymer Modification by in Situ Phototriggered Deprotection and Thiol-Ene Chemistry. Polym. Chem. 2012, 3 (7), 1740-1749.

(66) Biffinger, J. C.; Kim, H. W.; DiMagno, S. G. The Polar Hydrophobicity of Fluorinated Compounds. ChemBioChem 2004, 5 (5), 622-627.

(67) Riedel, M.; Stadermann, J.; Komber, H.; Simon, F.; Voit, B. Synthesis, Post-Modification and SelfAssembled Thin Films of Pentafluorostyrene Containing Block Copolymers. Eur. Polym. J. 2011, 47 (4), 675-684.

(68) Pospiech, D.; Jehnichen, D.; Eckstein, K.; Scheibe, P.; Komber, H.; Sahre, K.; Janke, A.; Reuter, 
U.; Häußler, L.; Schellkopf, L.; et al. Semifluorinated PMMA Block Copolymers: Synthesis, Nanostructure, and Thin Film Properties. Macromol. Chem. Phys. 2017, 218 (18), 1-14.

(69) Noy, J.-M.; Koldevitz, M.; Roth, P. J. Thiol-Reactive Functional Poly(Meth)Acrylates: Multicomponent Monomer Synthesis, RAFT (Co)Polymerization and Highly Efficient Thiol- ParaFluoro Postpolymerization Modification. Polym. Chem. 2014, 6, 436-447.

(70) Cavalli, F.; Mutlu, H.; Steinmueller, S. O.; Barner, L. The Para-Fluoro-Thiol Reaction as a Powerful Tool for Precision Network Synthesis. Polym. Chem. 2017, 8 (25), 3778-3782.

(71) Varadharajan, D.; Delaittre, G. Accessing Libraries of Bifunctional Block Copolymers Using Distinct Pentafluorophenyl Moieties. Polym. Chem. 2016, 7, 7488-7499.

(72) Delaittre, G.; Barner, L. The Para-Fluoro-Thiol Reaction as an Efficient Tool in Polymer Chemistry. Polym. Chem. 2018, 9, 2679-2684.

(73) Turgut, H.; Delaittre, G. On the Orthogonality of Two Thiol-Based Modular Ligations. Chem. - A Eur. J. 2016, 22 (4), 1511-1521.

(74) Bhagat, S. D.; Chatterjee, J.; Chen, B.; Stiegman, A. E. High Refractive Index Polymers Based on Thiol-Ene Cross-Linking Using Polarizable Inorganic/Organic Monomers. Macromolecules 2012, 45 (3), 1174-1181.

(75) Junkers, T.; Barner-Kowollik, C. The Role of Mid-Chain Radicals in Acrylate Free Radical Polymerization: Branching and Scission. J. Polym. Sci. Part A Polym. Chem. 2008, 46 (23), 75857605.

(76) Vandenbergh, J.; Reekmans, G.; Adriaensens, P.; Junkers, T. Synthesis of Sequence Controlled Acrylate Oligomers via Consecutive RAFT Monomer Additions. Chem. Commun. 2013, 49 (88), 10358.

(77) Pomposo, J. A.; Perez-Baena, I.; Buruaga, L.; Alegría, A.; Moreno, A. J.; Colmenero, J. On the Apparent SEC Molecular Weight and Polydispersity Reduction upon Intramolecular Collapse of Polydisperse Chains to Unimolecular Nanoparticles. Macromolecules 2011, 44 (21), 8644-8649.

(78) Laguna, M. T. R.; Medrano, R.; Plana, M. P.; Tarazona, M. P. Polymer Characterization by SizeExclusion Chromatography with Multiple Detection. J. Chromatogr. A 2001, 919 (1), 13-19.

(79) Sanchez-Sanchez, A.; Asenjo-Sanz, I.; Buruaga, L.; Pomposo, J. A. Naked and Self-Clickable Propargylic-Decorated Single-Chain Nanoparticle Precursors via Redox-Initiated RAFT Polymerization. Macromol. Rapid Commun. 2012, 33 (15), 1262-1267.

(80) Blasco, E.; Tuten, B. T.; Frisch, H.; Lederer, A.; Barner-Kowollik, C. Characterizing Single Chain Nanoparticles (SCNPs): A Critical Survey. Polym. Chem. 2017, 8 (38), 5845-5851.

(81) Wyatt, P. J. Measurement of Special Nanoparticle Structures by Light Scattering. Anal. Chem. 2014, 86 (15), 7171-7183.

(82) Pethrick, R. A. Polymers and Neutron Scattering. Polym. Int. 1995, 37 (2), 149-150.

(83) Pahnke, K.; Brandt, J.; Grynova, G.; Lindner, P.; Schweins, R.; Schmidt, F. G.; Lederer, A.; Coote, M. L.; Barner-Kowollik, C. Entropy Driven Chain Effects on Ligation Chemistry. Chem. Sci. 2015, $6(2), 1061-1074$.

(84) Zimm, B. H. The Scattering of Light and the Radial Distribution Function of High Polymer Solutions. J. Chem. Phys. 1948, 16 (12), 1093-1099.

(85) Galinsky, G.; Burchard, W. Starch Fractions as Examples for Nonrandomly Branched Macromolecules. 2. Behavior in the Semidilute Region. Macromolecules 1996, 29 (5), 1498-1506.

(86) SasView.org. SasView for Small Angle Scattering Analysis http://www.sasview.org/ (accessed Jan 19, 2020).

(87) Lederer, A.; Burchard, W.; Hartmann, T.; Haataja, J. S.; Houbenov, N.; Janke, A.; Friedel, P.; Schweins, R.; Lindner, P. Dendronized Hyperbranched Macromolecules: Soft Matter with a Novel Type of Segmental Distribution. Angew. Chemie - Int. Ed. 2015, 54 (43), 12578-12583. 
(88) Lederer, A.; Burchard, W.; Khalyavina, A.; Lindner, P.; Schweins, R. Is the Universal Law Valid for Branched Polymers? Angew. Chemie Int. Ed. 2013, 52 (17), 4659-4663.

(89) Dockhorn, R.; Plüschke, L.; Geisler, M.; Zessin, J.; Lindner, P.; Mundil, R.; Merna, J.; Sommer, J.U.; Lederer, A. Polyolefins Formed by Chain Walking Catalysis-A Matter of Branching Density Only? J. Am. Chem. Soc. 2019, 141 (39), 15586-15596.

(90) Tande, B. M.; Wagner, N. J.; Mackay, M. E.; Hawker, C. J.; Jeong, M. Viscosimetric, Hydrodynamic, and Conformational Properties of Dendrimers and Dendrons. Macromolecules 2001, 34 (24), 8580-8585.

(91) Zimm, B. H.; Stockmayer, W. H. The Dimensions of Chain Molecules Containing Branches and Rings. J. Chem. Phys. 1949, 17 (12), 1301-1314.

(92) Stockmayer, W. H.; Fixman, M. Dilute Solutions of Branched Polymers. Ann. N. Y. Acad. Sci. 1953, 57 (4), 334-352.

(93) Glatter, O.; Kratky, O. Small Angle X-Ray Scattering; Academic Press, 1982.

(94) Casassa, E. F. Light Scattering from Very Long Rod-Like Particles and an Application to Polymerized Fibrinogen. J. Chem. Phys. 1955, 23 (3), 596-597.

(95) Marion, J.; Trovaslet, M.; Martinez, N.; Masson, P.; Schweins, R.; Nachon, F.; Trapp, M.; Peters, J. Pressure-Induced Molten Globule State of Human Acetylcholinesterase: Structural and Dynamical Changes Monitored by Neutron Scattering. Phys. Chem. Chem. Phys. 2015, 17 (5), 3157-3163.

(96) Burchard, W. Light Scattering from Polysaccharides as Soft Materials. In Soft Matter Characterization; Borsali, R., Pecora, R., Eds.; Springer Netherlands: Dordrecht, 2008; pp 463603.

(97) Receveur-Brechot, V.; Durand, D. How Random Are Intrinsically Disordered Proteins? A Small Angle Scattering Perspective. Curr. Protein Pept. Sci. 2012, 13 (1), 55-75.

(98) Pedersen, J. S.; Schurtenberger, P. Scattering Functions of Semiflexible Polymers with and without Excluded Volume Effects. Macromolecules 1996, 29 (23), 7602-7612.

(99) Frisch, H.; Kodura, D.; Bloesser, F. R.; Michalek, L.; Barner-Kowollik, C. Wavelength-Selective Folding of Single Polymer Chains with Different Colors of Visible Light. Macromol. Rapid Commun. 2019, 1900414, 1-6.

(100) Moreno, A. J.; Bacova, P.; Verso, F. Lo; Arbe, A.; Colmenero, J.; Pomposo, J. A. Effect of Chain Stiffness on the Structure of Single-Chain Polymer Nanoparticles. J. Phys. Condens. Matter 2017, 30 (3), 34001. 
Table of Contents

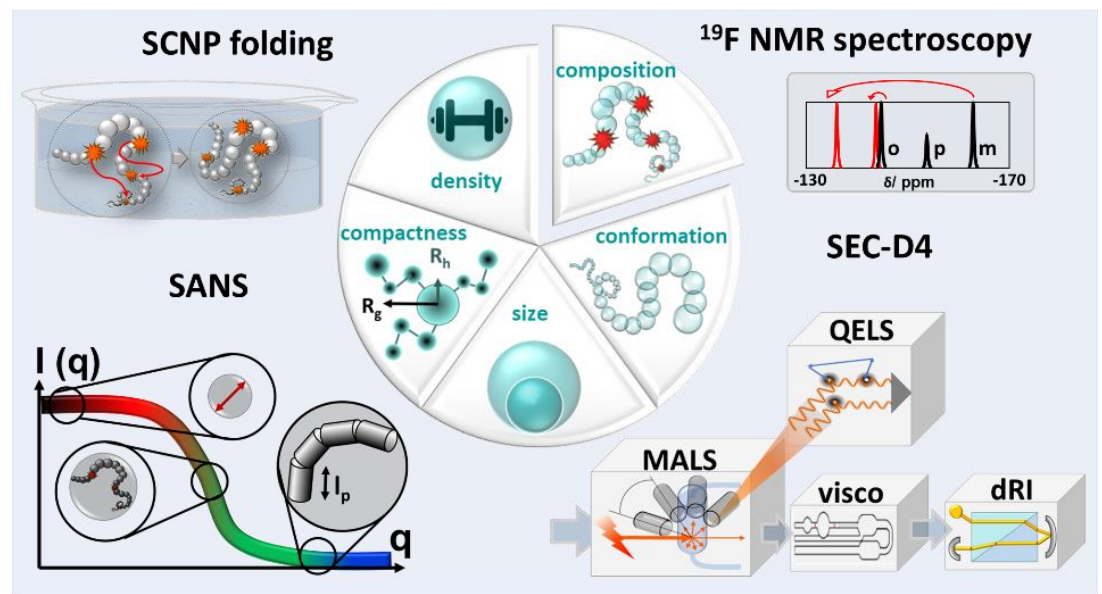

